
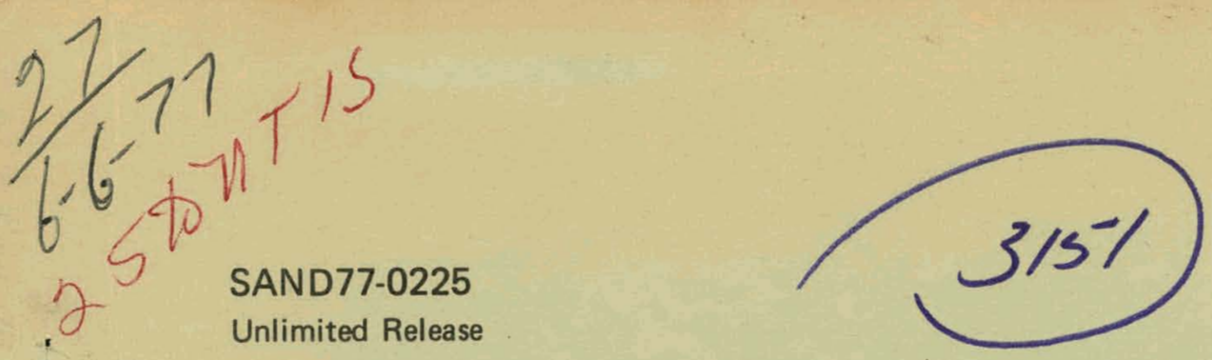

\title{
Digital Spectral Analysis and Filtering of Experimental Aerodynamic Data
}

Edward L. Clark, Jr., Robert H. Croll, Jr.

Prepared by Sandia Laboratories, A lbuquerque, New Mexico 87115

and Livermore, California 94550 for the United States Energy R esearch

and Development Administration under Contract AT (29-1)-789

Printed May 1977 


\section{DISCLAIMER}

This report was prepared as an account of work sponsored by an agency of the United States Government. Neither the United States Government nor any agency Thereof, nor any of their employees, makes any warranty, express or implied, or assumes any legal liability or responsibility for the accuracy, completeness, or usefulness of any information, apparatus, product, or process disclosed, or represents that its use would not infringe privately owned rights. Reference herein to any specific commercial product, process, or service by trade name, trademark, manufacturer, or otherwise does not necessarily constitute or imply its endorsement, recommendation, or favoring by the United States Government or any agency thereof. The views and opinions of authors expressed herein do not necessarily state or reflect those of the United States Government or any agency thereof. 


\section{DISCLAIMER}

Portions of this document may be illegible in electronic image products. Images are produced from the best available original document. 


\section{PAGES 1 to 2 WERE INTENTIONALLY LEFT BLANK}


SAND77-0225

Unlimited Release

Printed May 1977

\section{DIGITAL SPECTRAL ANALYSIS AND FILTERING OF EXPERIMENTAL AERODYNAMIC DATA}

E. L. Clark, Jr. and R. H. Croll, Jr. Experimental Aerodynamics Division 1334 Sandia Laboratories Albuquerque, New Mexico 87115

\section{ABSTRACT}

Digital frequency analysis techniques are used to determine the frequency content of a set of time-dependent data and to improve the quality of the data by eliminating undesired frequencies. This report provides an introduction to the use of these teclinfques with nonrandom data. Included are guidelines for acquisition and processing of data, calculation and interpretation of digital spectral estimates, and application of digital filters. The techniques are demonstrated by use of three examples derived from wind-tunnel data: (1) frequency analysis of 3-DOF angular motion data; (2) elimination of extraneous frequencies in dynamic stability data; and (3) determination of control surface forcing function frequencies.

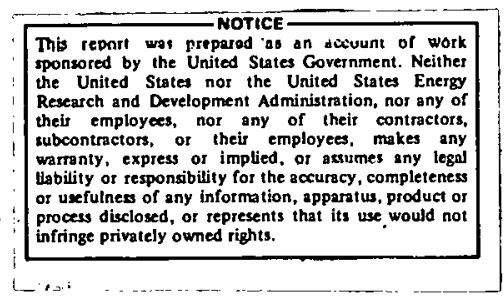




\section{ACKNOWLEDGMENT}

The authors wish to acknowledge the assistance of R. E. Jones, Applied Mathematics Division, Sandia Laboratories, who provided the fast Fourier transform FORTRAN routines used in this study and advised the authors on the use of these routines. D. R. MacKenzie and E. A. Koenig, Experimental Aerodynamics Division, Sandia Laboratories, assisted in the acquisition and processing of the data presented in this report. 
Nomenclature

Introduction . 9

The Fourier Transform : : 10

Data Acquisition and Processing . 15

Digital Spectral Analysis . . . . . $\quad 22$

Digital Filters . . . . 37

Verification of Procedures $\quad \therefore \quad 44$

Selected Applications . . 46

Frequency Analysis of Three-Degree-of-Freedom
Angular Motions

Elimination of Extraneous Frequencies in 50

Determination of Control Surface Forcing
Function Frequencies

Concluding Remarks . . . 56

References. $\quad: \quad 57$

ILLUSTRATIONS

Figure

Page

1 Fourier transform of a real function 13

2 Procedure for digital frequency analysis : 16

3 Frequency aliasing in sampled data ... 20

4 Examples of correctly and incorrectly . . 21

5. Aliasing error in digital spectra $\quad 24$

6 Leakage error in digital spectra 25

7 Data wiinluws . $\quad \cdots 28$

8 Effect of Hanning window on leakage. 29

9 Spectra of data sets extended with $2048-\mathrm{N} .33$

10 Sample results for the Martin-Graham filter 42

11 Aliasing error in digital filters 43

12 Simulated tricyclic motion data 48

13 Dynamic stability data with support 51

14 Control surface dynamics 55 


\section{Nomenc lature}

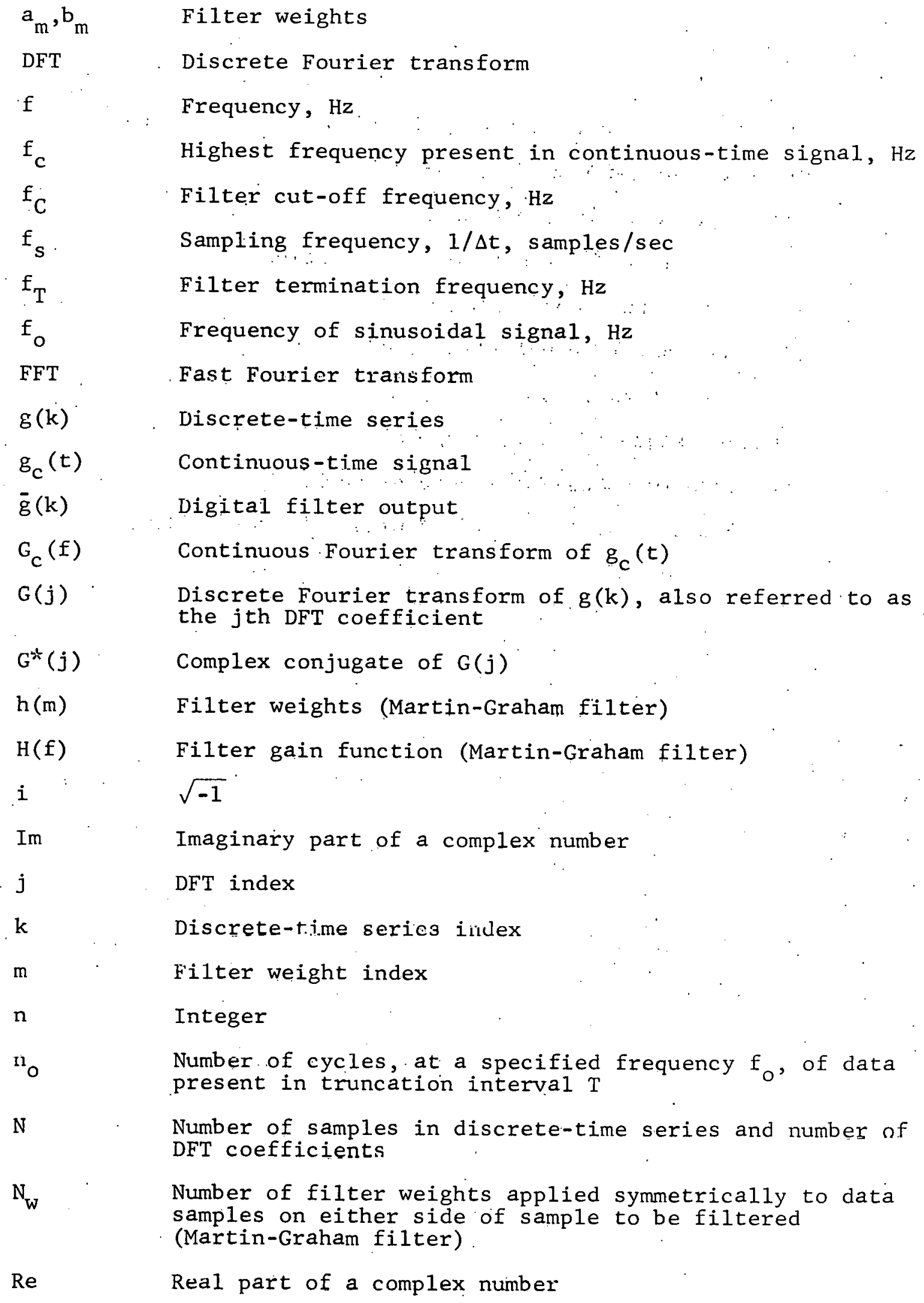

Re Real part of a complex number 


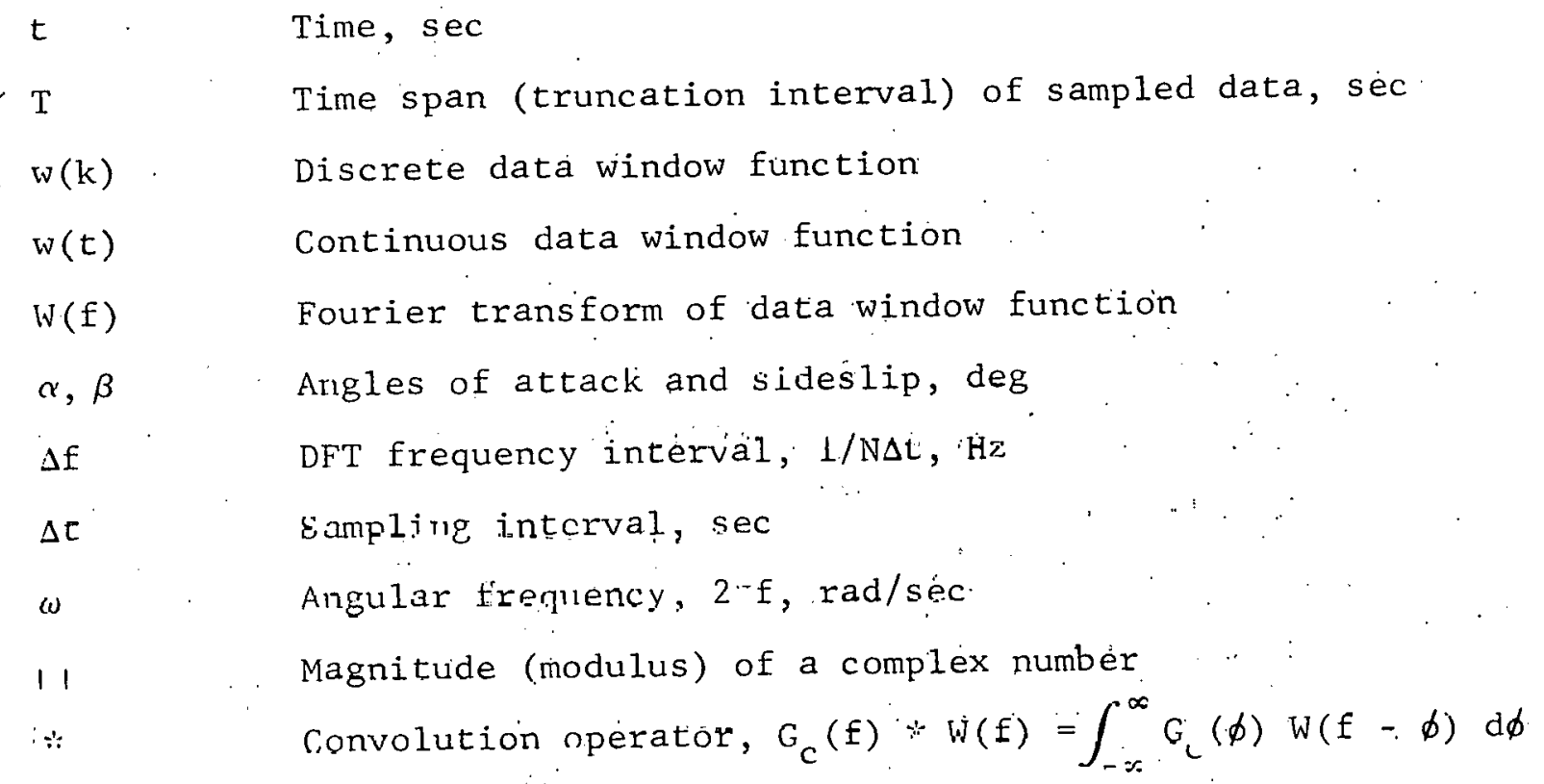


DIGITAL SPECTRAL ANALYSIS AND FILTERING

OF EXPERIMENTAL AERODYNAMIC DATA

\section{Introduction}

Spectral analysis provides an estimate of the frequency content for a set of time-dependent data. Filtering permits the attenuation of undesired frequencies in the data set. For many years, application of these techniques was limited to the use of electronic spectrum analyzers and filters. Unfortunately, these devices could not always satisfy data processing requirements and were expensive. Early digital techniques required unacceptable amounts of computer time. These problems prevented widespread usage of frequencydomain techniques.

In 1965, a major advancement. in digital frequency analysis occurred when the fast Fourier transform (FFT) was implemented. This computational procedure greatly reduces the time required to calculate discrete Fourier transforms. Continuing improvements in computer hardware, combined with availability of the FFT, have made digital spectral analysis and filtering practical. These techniques are being increasingly applied in science and engineering fields. In experimental aerodynamics, digital frequency analysis has been used in some investigations of turbulence and unsteady pressures. These are applications which involve random signals, that is, the instantaneouo magnitude of the signal cannot be specified for any given instant of time. The use of these techniques with nonrandom signals,*

*Nonrandom signals are also called deterministic and can be represented mathematically as functions of a continuous variable. Repetition of the physical process that generated the signal, under essentially identical conditions, would yield essentially. the same signal. Random signals are frequently referred to as noise-like signals and cannot be described by a continuous mathematical function. Repetition of the physical process, under essentially identical conditions, would yield signals which would be simllar only in their statistical properties. 
wherein the instantaneous magnitude is specified at any instant, has been very limited in experimental aerodynamics.

The purpose of this report is to provide an introduction to the use of digital frequency analysis methods with nonrandom signals. The principal type of nonrandom, time-dependent data encountered in experimental aerodynamics is that obtained in dynamic stability tests. It will be shown, by examples, that the analysis of dynamic data can be significantly improved through application of these techniques. The basic concepts of frequencydomain techniques will be presented without nathematical derivations or proofs. The reader will find mathematical developments in many of the cited references.

\section{The Fouriex Transform}

The basis of digital frequency analysis is the Fourier transform which is used in both the continuous and discrete forms. Given a continuous function in the time domain, $g_{c}(t)$, the corresponding continuous function in the frequency domain, $G_{C}(f)$, is given by the continuous Fourier transform,

$$
G_{c}(f)=\int_{-\infty}^{\infty} g_{C}(t) e^{-i 2 i t t} d t \quad,
$$

where $i=\sqrt{-1}$. Likewise, a function in the frequency domain can be transformed to the time domain by use of the rrverse Fourier transform,

$$
g_{c}(t)=\int_{-\infty}^{\infty} G_{c}(f) e^{i 2 i i f t} d f
$$

When the functions $g_{c}(t)$ and $G_{c}(f)$ are related by Eqs. (1) and (2), the two functions are termed a Fourler transform pair.* Throughout this report, reference will be made to a few of the more frequently encouncered Furier

*Definition of the continuous Fourier transform pair is not uniform in the literature, Other definitions differ from Eqs. (1) and (2) by constant factors. 1 Similar lack of uniformity exists for the discrete Fourier transform pair.2,3 As long as the definitions are consistent for the transform pair, this lack of uniformity is not a problem. 
transform pairs. Also, several basic Fourier transform properties will be used. These transform pairs and transform properties are described in detail in References 4 and 5. There are mathematical functions for which the Fourier transform does not exist. However, if the function to be transformed describes a physical quantity, the existence of its transform may be assumed. Physical possibility is a sufficient condition for the existence of a transform. 6

For a finite, sampled set of data, the discrete Fourier transform (DFT) pair must be used,

$$
G(j)=\sum_{k=0}^{N-1} g(k) e^{-i 2 \pi j k / N} \quad ; \quad j=0,1, \cdots, N-1
$$

and

$$
g(k)=\frac{1}{N} \sum_{j=0}^{N-1} G(j) e^{i 2 \pi j k / N} ; \quad k=0,1, \cdots, N-1,
$$

where $\mathrm{N}$ is the number of samples in the discrete-time series, $\mathrm{g}(\mathrm{k})$. The discrete values of time, $t(k)$, at which the discrete-time series is defined are given by

$$
t(k)=k \Delta t \quad ; \quad k=0,1, \cdots, N-i,
$$

where $\Delta t$ is the sampling interval of the data, that is, the time interval between consecutive samples. The disclele frequencies, $f(j)$, corresponding to the DFT coefficients, $G(j)$, are given by

$$
f(j)=j \Delta f \quad ; \quad j=0,1, \cdots, N-1 .
$$

where

$$
\Delta \mathrm{f}=1 / \mathrm{N} \Delta \mathrm{t}
$$


If the DFT pair is used to approximate the continuous Fourier transform pair, the right side of Eq. (3) must be multiplied by $\Delta t$ and the right side of Eq. (4) must be multiplied by $N \Delta f(=1 / \Delta t)$.

In most applications the time series $g(k)$ will be real. However, in the frequency domain, the DFT coefficients, $G(j)$, will generally be complex. If the time series is real, a redundancy occurs in the DFT coefficients since, for $1 \because \mathrm{j} \quad(\mathrm{N}-1)$,

$$
G(N-j)=G^{*}(j)
$$

where $G^{*}(j)$ is the complux ionjugate of $G(j)$. I'herefure, the traneform of any real function has conjugate symmetry (that is, the real part of the transform is an even function and the imaginary parl is ain odd function) about $j=0$ and $j=N / 2$, corresponding to the frequencies $f=U$ and $f=1 / 2 \Delta t$, respectively. Thus, only the $1+N / 2$ coefficlenLs $G(0)$ through $G(N / 2)$ are needed to completely define the DFT. l'he frequency, $f-1 / 2 \Delta t$, plays an important role in sampled data and is thoroughly discussed in the next section.

A typical redl function and its transform are shown in figure 1. The The continuous function $g_{C}=e^{-t}$ was sampled 32 times with a sampling interval of $0.25 \mathrm{sec}$ for this example. Tf the sampled function $\mathrm{g}(\mathrm{k})$ is defined at a discontinuity as having the average value, as shown in Figure la at $t=0$, the DFI will more closely approximate the continuous transform. For the continuous transform, $G_{C}(t)$, and the discrete transform, $G(j)$, (shown in Figure 1b) Re and Im denote the real and imaginary parts, respertively. For equivalence belwetu the continuous find discrete transforms, the DFT coefficients [defined by Eq. (3)] have been lultiplicd by $\Delta t$. This figure clearly shows Lhe conjugate symmetry of the DFT-Re G(j) has even symmetry about $j=\mathrm{j} / 2=16$, and $\operatorname{Im~} G(j)$ has odd symmetry about the same point. Conventionally, the DFT coefficients for $j>N / 2$ are associated with a negutive frequency, $f=-(N-j) \Delta f$, as shown. in the figure. In this example, the 

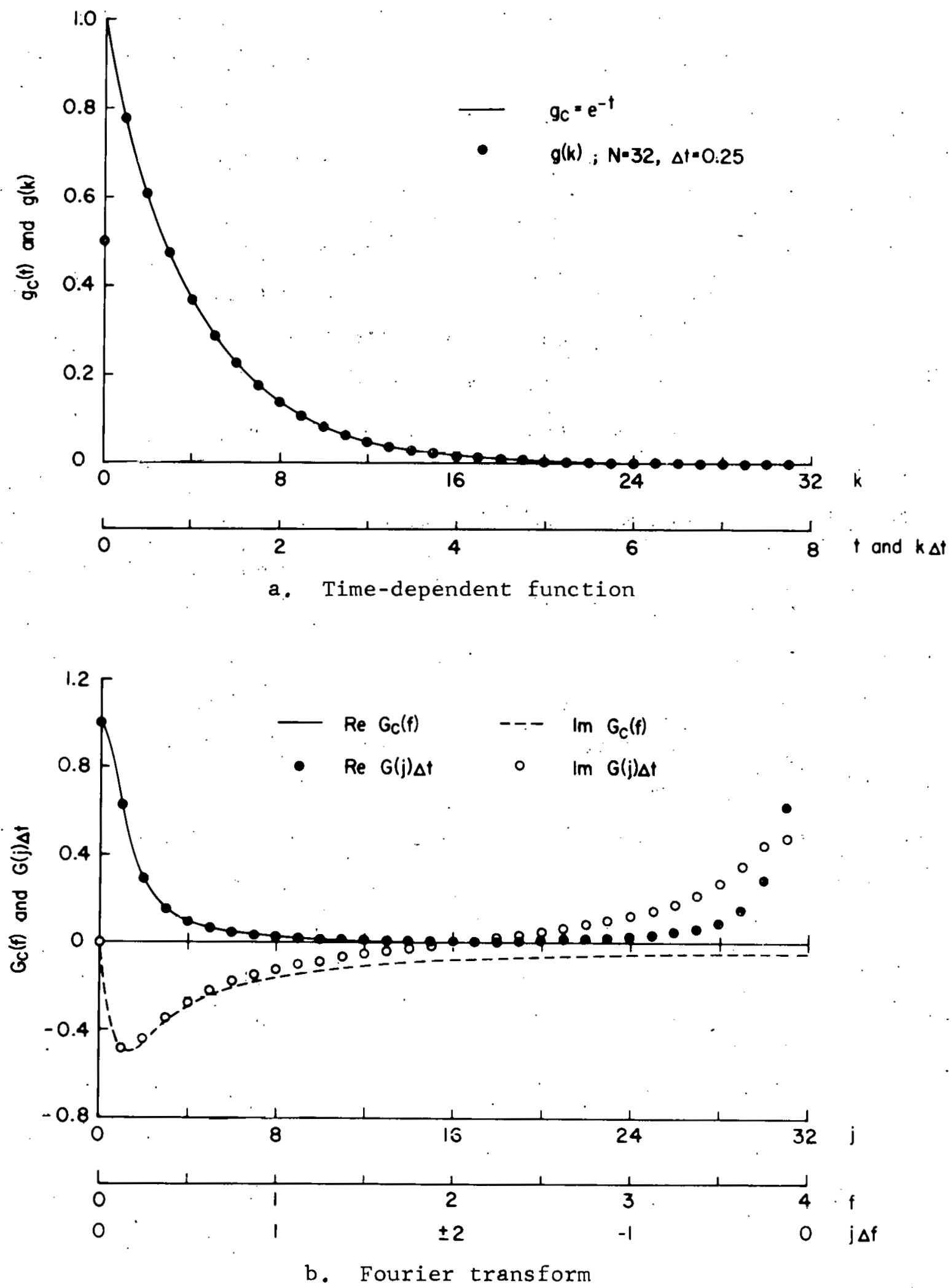

Figure 1. Fourier transform of a real function 
DFT is a poor approximation of the imaginary part of the continuous transform. Increasing $\mathrm{N}$ and decreasing $\Delta t$ (so that the total time $N \Delta t$ is constant) will greatly improve the agreement between the two transforms. With $\mathrm{N}=256$ and $\Delta t=0.03125 \mathrm{sec}$, the difference is approximately 1.3 percent at $\mathrm{f}=2 \mathrm{~Hz}$.

The discrete Fourier transform is of interest primarily because it provides an approximation to the continuous Fourier transform that is suitable for digital computation. However, it is essential that the user be aware of the differences in the two transforms and the errors that arise from the approximation. To permit digital computation, both the time and frequency functions must be sampled. A direct consequence of samplitry is that periodicity is implied in both the t1me and frequency domains. Brigham ${ }^{7}$ points out, "If one will always remember that the $N$ sample values of the time-domain function represent one sample of a peitiodic function, then application of the discrete Fourier transform shuuld result in fow surprises." Furthermore, truncation of the time function to provide a finite number of samples introduces errors in the DFT. There is only one class of waveforms for which the discrete and continuous fourier transforms are exactly the same-periodic, frequency-limited waveforms with a truncation interval, $T=N \Delta t$, equal to exactly an integer multiple of the waveform period. This class of waveforms is very limited, being restricted to signals which have a single frequency or a fundamental frequency with harmonics (integral multiples of the fundamental). Fortunately, acceptable agreement between discrete and continuous fuurier transforms ran usually be obtäned fur llute gencral waveforma hy use of the techniques discussed in the following sections.

Direct computation of the DFT by Eq. (3) is uneconomical. However, the use of fast Fourier transform (FFT) algorithms permits practical calculation of the DFT coefficients. Although schemes for optimal calculatiun UF DFT's wcre published as early as $1903,{ }^{8}$ application of such a technique 
to digital computers was apparently overlooked for many years. In 1965, Cooley and Tukey, ${ }^{9}$ without knowledge of the earlier efforts, developed and programmed an efficient algorithm for computation of DFT's. This method, which is now known as the "fast Fourier transform" (FFT), requires about: $\mathrm{nN} / 2$ complex multiplications to evaluate the DFT coefficients associated with $\mathrm{N}=2^{\mathrm{n}}$ data samples. By contrast, direct computation of the coefficients requires about $\mathrm{N}^{2}$ multiplications. For $\mathrm{N}=2^{11}=2048$, calculation of the DFT by use of the FFT requires less than 1/370 of the time required for direct calculations.* Furthermore, as a result of the fewer operations, the FFT substantially reduces round-off errors associated with the computation. Programs that calculate fast Fourier trạs forms are widely available (e.g., Ref. 10, 11, 12, 13). The FORTRAN routines used in this study were FOURT $^{14}$ and FOUR $1^{14}$ with modifications for more efficient transformation of real data.

Data Acquisition and Processing

Most time-dependent data will be analog and must be sampled if digital frequency analysis techniques are to be used. The quality of the results obtained from either digital spectral analysis or filtering of sampled data is very dependent upon proper data acquisition and processing. The necessary procedures are described in this section and summarized in Figure 2.

The first step in processing the data (Figure 2, block 1) is to record the continuous signal with a wideband analog tape or disc recorder. This is a wise precaution in any case, and is imperative if the bandwidth

*Actual calculations, with a set of reall datán on a CDC 6600 computer with subroutine FOURT14 and on a Honeywe11 DDP-116 computer . with subroutine FOUR114 gave relative computation times (FFT: direct calculation) of $1: 753$ and $1: 472$, respectively, for $N=2048$. 
DATA ACQUISITION ANO PROCESSING

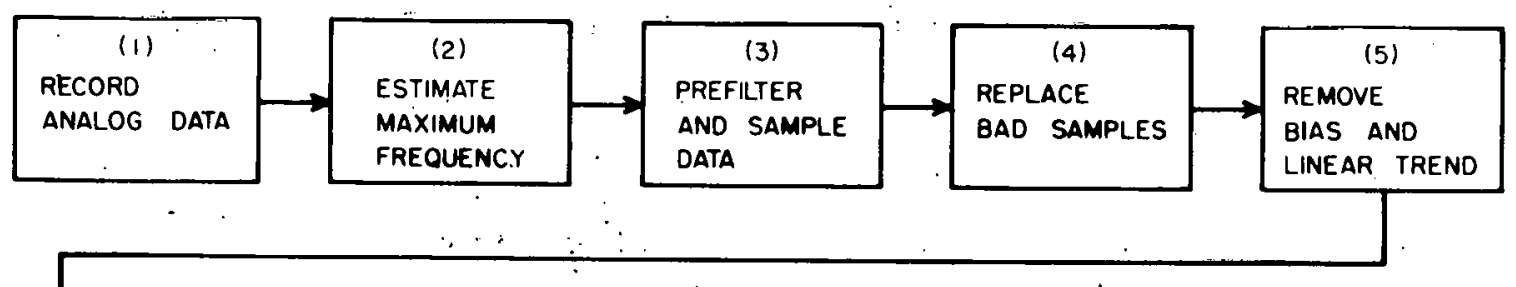

SPECTRAL ANALYSIS
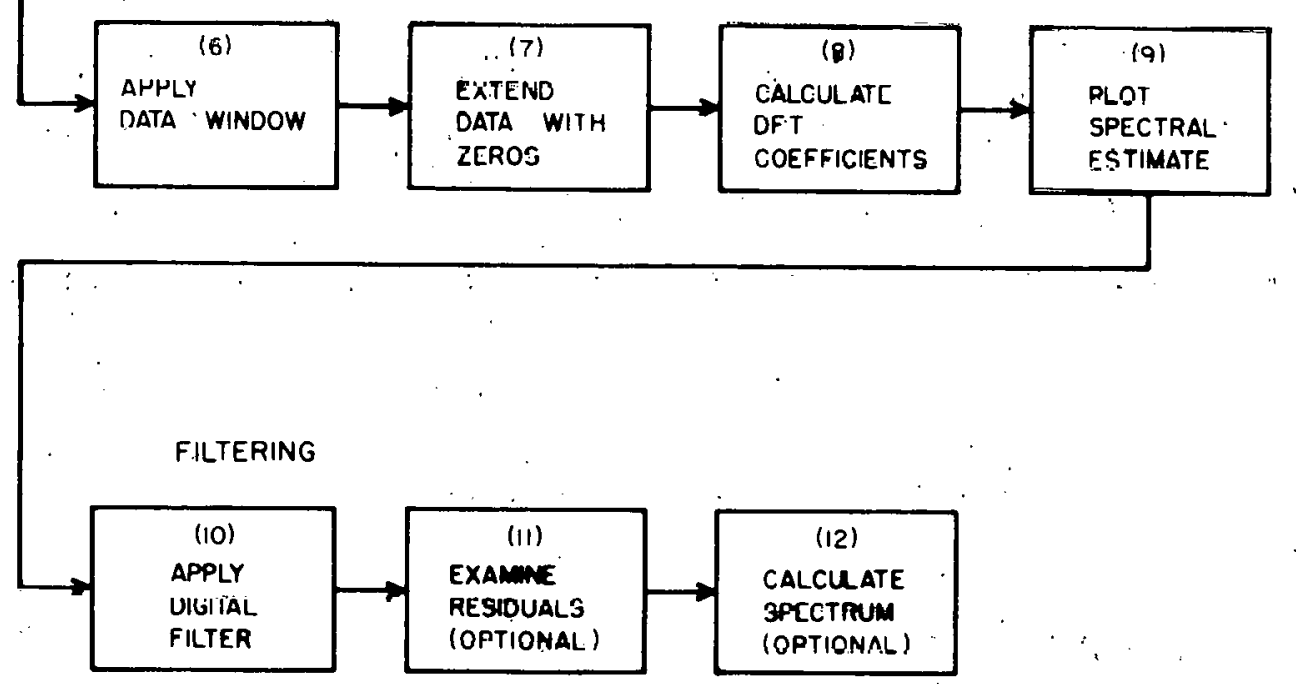

Figure 2. Procedure for digital frequency allalysis

(i.e., the frequency range) 'of the data is unknown. If the data are recorded, the approximate bandwidth "can be determined prior to sampling. Furthermore, it may be necessary' to sample the data more than once, usirly different sampling intervals and/or filter cut-off frequencies. Finally, most analog recorders have several record/playback speeds, permitting increased versatility in the data processing. The second step (Figure 2, block 2), is to display representative data on an oscillograph or oscilloscope. This permits an approximate evaluation of the highest significant 
frequency present in the data for subsequent use in determining the prefiltering and sampling parameters.

The most critical step in processing the data is the analog-to-digital conversion, that is, sampling the data (Figure 2, block 3). At this point, the analog signal is electronically prefiltered and passed through an analog-to-digital converter (ADC). The maximum signal level to be input to the ADC must be adjusted to remain within the amplitude range of the converter while allowing, as nearly as is practical, the full resolution of the converter. Otherwise, the discrete data will be distorted or excessively grainy. It is assumed in this discussion that the data are sampled uniformly, that is, with a constant sampling interval, $\Delta t$. Correct selection of the sampling interval is essential. Three criteria must be considered. The first, and most important, criterion is that $\Delta t$ must satisfy the sampling theorem:

If the Fourier transform, $G_{c}(f)$, of a continuous function $g_{C}(t)$ is zero for all frequencies greater than some frequency $f_{c}$, then the continuous function can be uniquely determined from its sampled values $g(k)$ if the sampling interval is selected to satisfy.

$$
\Delta t<1 / 2 \mathrm{f}_{c}
$$

The sampling frequency; $\mathrm{f}_{\mathrm{s}}$, is given by

$$
\mathrm{f}_{\mathrm{s}}=1 / \Delta \mathrm{t}
$$

Substituting $\Delta t$ from Eq. (7) gives

$$
f_{S}>2 f_{c} .
$$

Thus, the sampling theorem states that no. loss of information is incurred through the process of uniform sampling. if the signal is sampled at a rate greater than twice the highest frequency, $f_{c}$, present in the continuous 
signal.* Signals of practical interest may have Fourier transforms for which there does not exist a finite value of $f_{c}$ such that $G_{c}(f)=0$ for $f \because f_{c}$. Equation (7) then gives $\Delta t=0$, which is clearly unrealistic. In practice, the signal's frequency content above the maximum frequency of interest is attenuated by use of a continuous (electronic) low-pass filter.** With knowledge of the filter characteristics, a frequency can be determined above which components of the filtered signal will have negligible amplitude. This frequency is then used as $f_{c}$ in Eq. (7) to determine the maximum allowable sampling interval. It is generally recommended that the signal be sampled at a rate that is 2,5 to 6 times the highest significant frequency; thus, the sampling interval should be $4 / 5$ to $1 / 3$ that given by Eq. (7). A second tactor in selecting $\Delta t$ is the desired resolution of the digi= tal spectrum. From Eq. (6) it is seen that to obtain a particular frequency resolution, $\Delta f$, with the $D F T$, it is necessary that

$$
\Delta t=1 / N \Delta f \quad \cdot
$$

For two signal components having equal amplitudes but different frequencies to appear as separate peaks in a digital spectrum, a minimum separation of three DFT coefficients (i.e., $3 \Delta f$ ) is needed. ${ }^{15}$. For detection of a small. amplitude component, having a froquongy noarly equal th that of a large: amplitude component, more separation is required. This must be considered in determining $\Delta t$ from $\mathrm{Eq}$. (10) if it is essential to distinguish between two components which have nearly equal frequencies. It should be noted that

*The number of samples, $n$, present in one cycle at the frequency $f_{c}$ is, $n=1 /\left(f_{c} \Delta t\right)$. Substituting $\Delta t$ from Eq. (7) gives $n>2$. Thus, for no loss of information, the highest frequency must be sampled at a rate that yields more than two samples per cycle.

** It is advisable to display representative filtered and unfiltered data on an oscillograph or oscilloscope to insure that the basic character of the data is not changed by the filter. 
increasing $\Delta t$ to provide a finer frequency resolution results in a reduction in the signal bandwidth. Thus', the sampling interval must be a compromise between the $\Delta t$ values given by Eq. (7) (reduced by a factor of $4 / 5$ to $1 / 3$ ) and Eq. (10). From Eq. (10), it can be seen that increasing the number of samples, $N$, with fixed frequency resolution will permit smaller values of $\Delta t$ and, hence, greater bandwidth. However, the number of samples is usually restricted by computer storage and computational time limitation. . If a signal is time-limited, that is, the total time span, $T$, of the data is bounded, the resolution of the digital spectrum is limited to $\Delta f=1 / \mathrm{T}$ and cannot be improved by changing $\Delta t$ and $N$. Examples of time-limited signals include data from intermitterï tèst facilities, free-flight data, and data from highly damped motions.

The third factor influencing selection of sampling interval is not based on frequency analysis criteria, but rather upon subsequent data reduction requirements. For example, with dynamic stability data, data reduction techniques for determining the motion amplitude may require many more samples per cycle than the minimum ( $>2$ ) required by the sampling theorem.

The process of unifömly sampling a continuous function of time can yield a significant source of error if the sampling theorem is violated, that is, if $\Delta t \geq 1 / 2 f_{c}$. "From the theorem it is seen that the frequency range of sampled data is zero to one-half the sampling frequency. The upper limit, $\mathrm{f}_{\mathrm{s}} / 2=1 / 2 \Delta t$, is called the folding frequency. If the continuous signal contains frequencies greater than the folding frequency, these frequencies have "aliases" at lower frequencies and are folded into the interval $\left[0, f_{s} / 2\right]$ by the sampling process. "It can be shown ${ }^{16}$ that the frequencies $n f_{S} \pm f_{O}$, where $n$ is an integer, will appear in the sampled data as the frequency $f_{0}$. This is shown schematically in Figure 3. In Figure 4a, a continuous signal with frequency $\mathrm{f}_{\mathrm{o}}=15 \mathrm{~Hz}$ is shown. If this wave is sampled with $\Delta t=0.01 \mathrm{sec}\left(f_{s}=100\right.$ samples $\left./ \mathrm{sec}\right)$ as shown, there will be 6.67 samples per cycle and the signal is correctly sampled with no aliasing. 


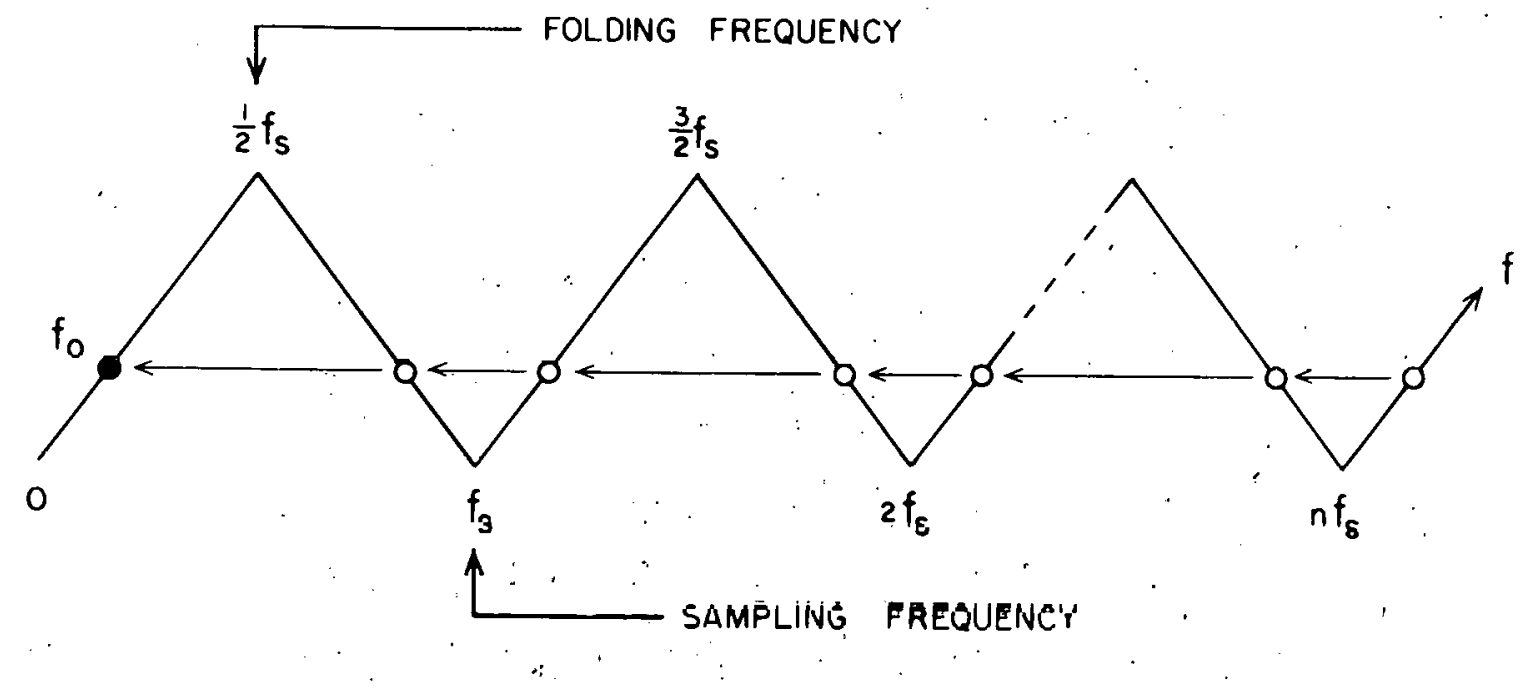

Figure 3: Frequency aliasing in sampled data

In Figure 4b, a continuous signal having a frequency equal to $f_{S}-f_{0}$ $(85 \mathrm{~Hz}$ ) is shown. If this signal is sampled with $\Delta t=0.01 \mathrm{sec}$ (corresponding to 1.18 samples per cycle), the frequency is aliased by $f_{0}$ (see Figurc 3) and the sampled data will appear the same as the samples from a $15 \mathrm{~Hz}$ signal with phase shifted $180 \mathrm{deg}$ from that shown in Figure $4 \mathrm{a}$. In l'igure $4 \mathrm{c}$, a continuous 'signal having a frequency equal to $\mathrm{f}_{\mathrm{s}}+\mathrm{f}_{\mathrm{o}}$ (115 $\mathrm{Hz}$ ) is shown. If this signal is sampled with $\Delta t=0.01 \mathrm{eec}$ (corresponding to 0.87 samples per cycle), the frequency is aliased by $f_{0}$ (see Figure 3 ) and "the sampled data will be identical to that obtained from the $15 \mathrm{~Hz}$ signal of Figure 4a. Therefore; it is obvious that trequencies greater than the folding frequicncy must be attenuated to an insignificant level by electronically filtering the continuous signal prior to sampling. It should be noted that photographic data, whether still photographs, as in a ballistic range test, or motion pictures, as in. a wind tunnel free-flight test, are subject to aliasing error and the guidelines given here for selecting $\Delta t$. apply to the time between pictures or the framing interval. 


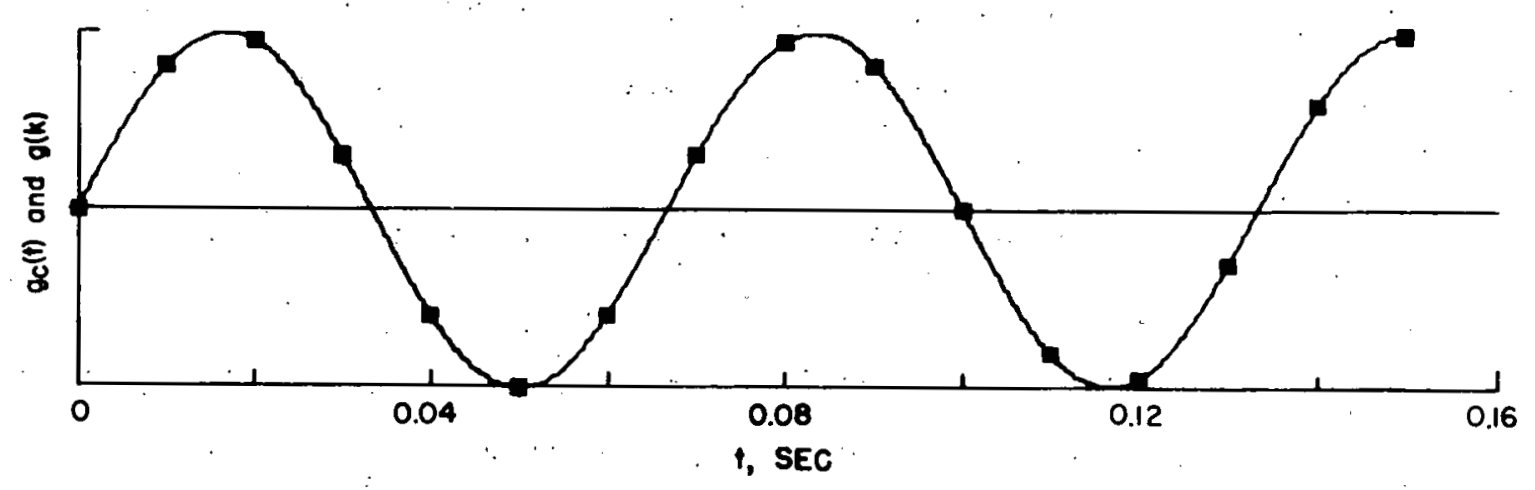

a. $f=f_{0}$, correctly sampled. $\left(f_{s}>2 f\right)$

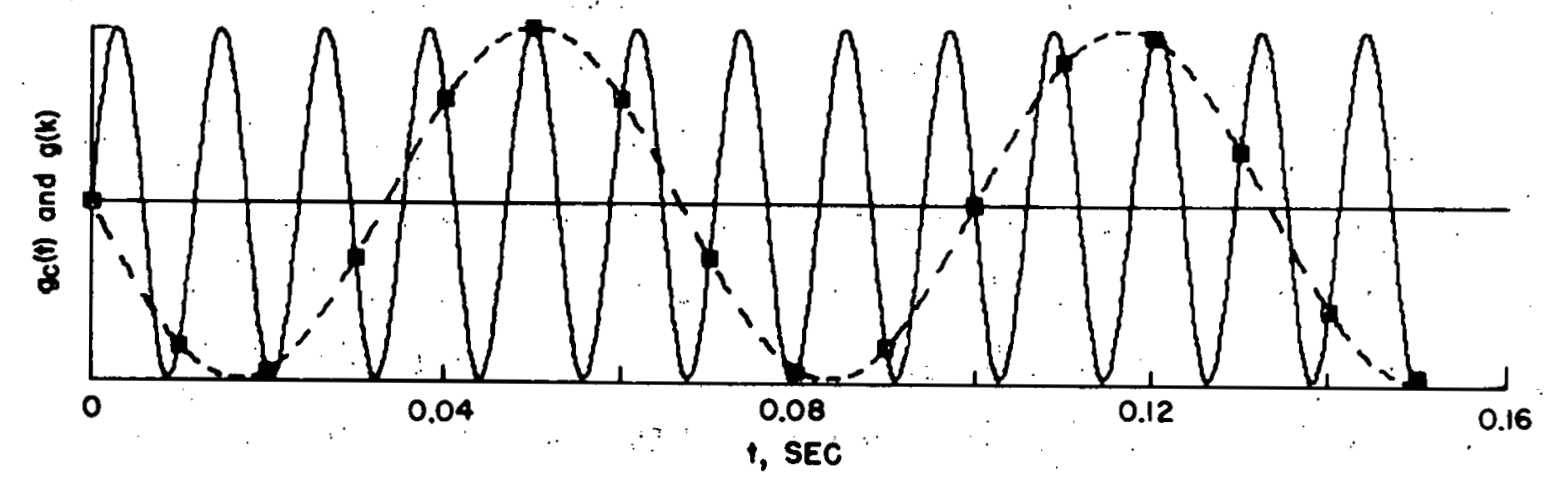

b. $\mathrm{f}=\mathrm{f}_{\mathrm{s}}-\mathrm{f}_{\mathrm{o}} ;$ incorrectly sampled $\left(\mathrm{f}_{\mathrm{s}}<2 \mathrm{f}\right)$

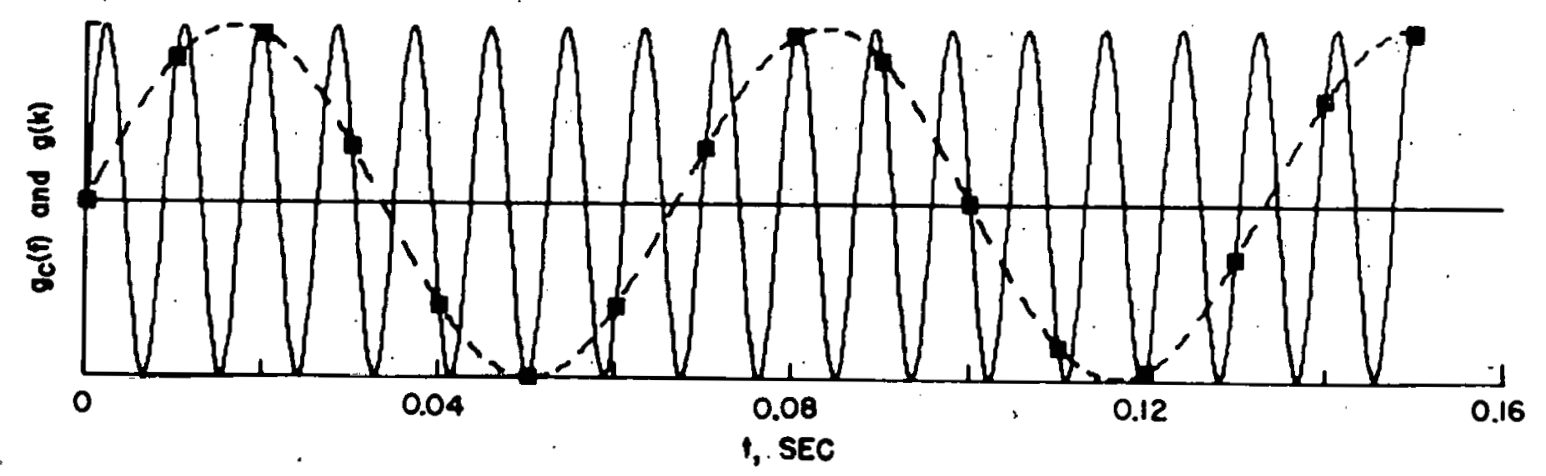

$$
\text { c. } f=f_{s}+f_{o} \text {, incorrectly sampled }\left(f_{s}<2 f\right)
$$

Figure 4. Examples of correctly and incorrectly sampled signals 
Aliasing can be used advantageously to increase the sampling frequency capability of the data system. Any frequency interval $\left[\mathrm{nf}_{\mathrm{s}} / 2,(\mathrm{n}+1) \mathrm{f}_{\mathrm{s}} / 2\right]$ can be selected for analysis (see Figure 3). The frcquency content of the continuous signal is limited to the desired interval by use of an electronic bandpass filter, and the sampling and analysis are done as for the interval $\left[0, f_{s} / 2\right]$. The upper frequency for which this technique can be used is limited only by the aperture time of the ADC. Further details on this technique are given in Ref. 15.

Two steps remain in processing the sampled data (Figure 2, blocks 4 and 5). First, any obviously bad data samples should be replaced by acceptable values. A bad data sample appears as an impulse (Dirac delta) function superimposed on the daca and can shift the magnitude of the digita1 spectrum over the entire frequency range. Second, if the data are biased, for example, as the result of instrument zero shifis, lhe dala slivuld be adjusted to have a zero mean by subtracting the data mean from each samplc. This operation reduces the magnitude of the first DFT coefficient, which corresponds to $f=0$, and avoids discontinuities in the data if zeros are added as described in the next section. In some cases it may also be necessary to remove any linear trend that the data might have, for example, as the result of instrument drift. Recalling that use of the DFT implies periodicity in the time-domain function, removal of any linear trend reduces the discontinuities which would otherwise occur at the end-points of the tinte series.

\section{Digital Spectral Analysis}

The purpose of calculating the digital spectrum is to provide an estimate of the frequency content of the data. This may be the final objective of the data analysis, or it may be an intermediate step prior to filtering and subsequent data reduction. 
The continuous Fourier transform of a continuous time-dependent signal will identify the different frequency components which are present in the signal. It is this property of the Fourier transform that makes the transform useful for spectral analysis. For example, the continuous Fourier transform of a composite wave consisting of an arbitrary number of sinusoidal components, each with a different frequency, is simply a series of impulse functions which occur at frequencies corresponding to the component frequencies. However, as was mentioned in the section on the Fourier transform, the DFT is only an approximation to the continuous Fourier transform (except for a limited class of waveforms which are of little practical interest). The differences between the continuous and discrete Fourier transform are the result of truncation of the time-domain function and aliasing of frequencies.

Aliasing was described in the previous section. The effect of aliasing on the DFT and hence, digital spectra, is shown in Figure 5. The continuous signal, $g_{c}(t)$, and its transform are shown in Figure $5 a$ where $\left|G_{C}(f)\right|$ is the magnitude of the continuous transform. Because the transform of a real function has conjugate symmetry, the transform magnitude is symmetrical about $f=0$. Thus, it is not necessary to show both sides of the transform magnitude; and only the side which corresponds to positive frequencies is presented in this and subsequent figures, Sampling the signal with a sampling interval $\Delta t$ causes the transfopm of the continuous function țo be replicated at intervals, $f_{s}=1 / \Delta t$, as shown in Figures Jl alld se. In Figure 5b, the sampling interval satisfies the sampling theorem and for the frequency region of interest, $0 \leq \mathrm{f} \leq \mathrm{f}_{\mathrm{s}} / 2$, the transform is identical with the transform of the continuous signal. The aliased transform shown in Figure 5e differs from the desired transform because the sampling theorem has been violated by using a sampling interval that is too large. This error can be reduced by band-limiting the signal (e.g., by prefiltering) and choosing a satisfactory sampling interval. 

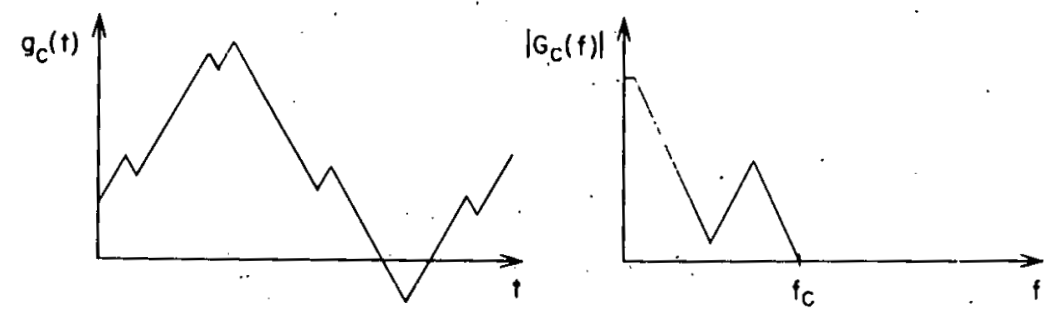

a. Continuous signal
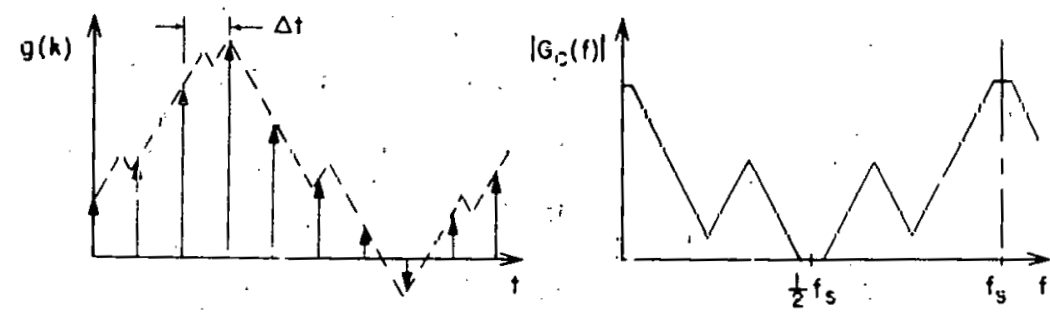

b. Sampled sifgnal, $\mathrm{f}_{s}>2 \underline{f}_{\mathrm{f}}$
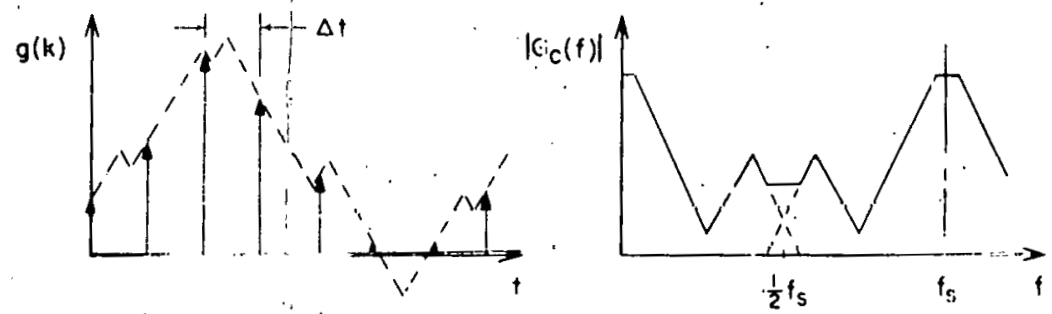

c. Sampled signal, $\Gamma_{s}<2 f_{c}$

Figure 5. Aliasing error in digital spertra

The second error, denoted as "leakage", is the result of timedomain truncation and occulrs because the number of samples mist be finite. This error is shown in Figure 6; where again the transform values for negative frequencies are riot shown. In Figure $6 a$, a cuntinuous signal $g_{c}(t)=\cos \left(2 i f_{0} t\right)$, and the magnitude of j.ts continuous transform are given. To obtain a finite lengtr: of signal, "we multiply the signal by a rectangular truncation. function or "wilndow" of length $\mathrm{T}=\mathrm{N} \Delta \mathrm{t}$. The window and the 


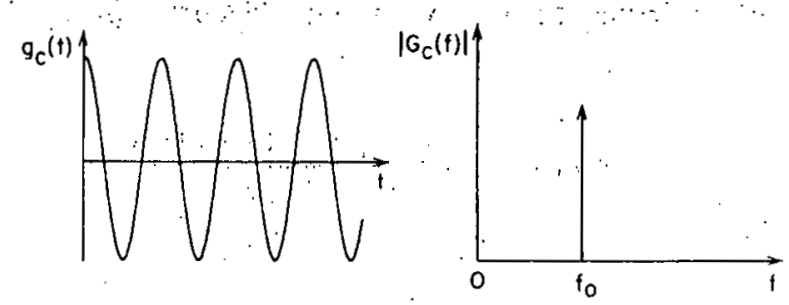

a. Continuous signail
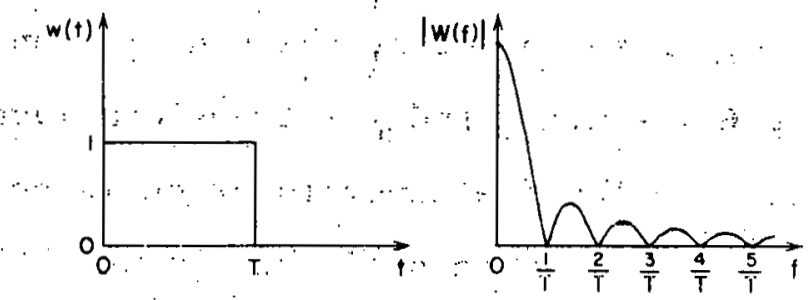

b. Truncation function (window)
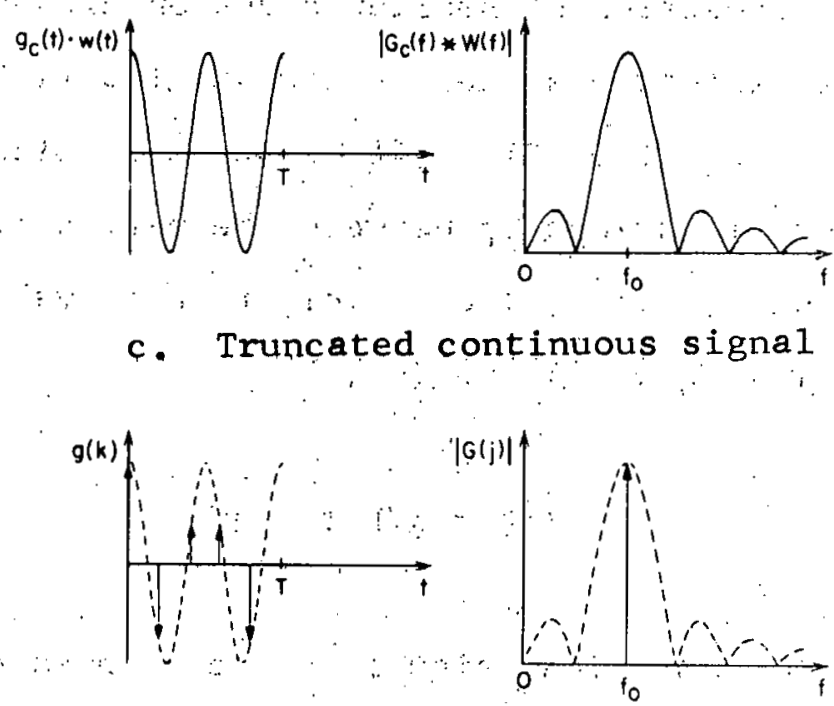

d. Sampled signal, T equal to period
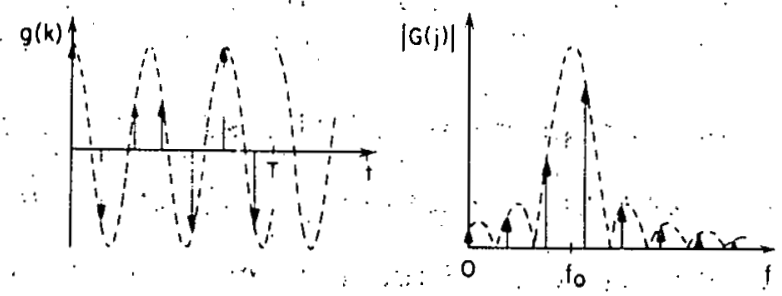

e. Sampled signal, T not equal to period

Figure 6. Leakage error in digital spectra 
magnitude of its transform are given in Figure $6 \mathrm{~b}$, where

$$
|W(f)|=\left|\frac{T \sin (\pi f T)}{\pi f T}\right|
$$

Multiplication in the time domain is equivalent to convolution in the frequency domain. Thus, while we want the transform $G_{c}(f)$; of the continuous signal $g_{c}(t)$, we are forced to accept the convolution $G_{c}(f) * W(f)$. The truncated signa $1, g_{c}(t): w(t)$, and ifs transform magnitude, $\left|G_{c}(f) * W(f)\right|$, are given in ligure $6 \mathrm{c}$. The frequency tunction is no longer a single impulse at $\ddot{H}_{n}$ but is now a continuous tunction which has its maximum value at $f_{o}$. The portion of this function which is centercl about $f_{0}$ is called the mainlobe, and the series of smalier peaks on either side are called sidelobes. If the truncated signal is now sampled (with $\Delta t$ selected to give negligible aliasing) and the DFT coefficients are calculated, the results will depend upon the relationship between the period of the signal and the truncation interval, T. The DFT coefficients are evaluated at the frequencies $f(j)$, where, from Eqs. (5) and (6);

$$
f(j)=j / N \Delta t=j / T
$$

'lhe frequency of the sinusoidal signal can be expressed as

$$
\mathrm{f}_{0}=\mathrm{n}_{0} / \mathrm{T}
$$

where $n_{0}$ is the number of periods of the signal in the truncation interval. If $n_{0}$ is an integer, the DFT frequency $f(j)$ for $j=n_{0}$ coincides exactly with the signal frequency $\mathrm{f}_{\mathrm{O}^{\circ}}$. Furthermore, the frequency interval, $\Delta \mathrm{f}=1 / \mathrm{T}$, at which the DFT coefficients are evaluated is equal to the spacing of the sidelobes (Figure $6 \mathrm{~b}$ or $6 \mathrm{c}$ ). Hence, all of the DFT coefficients except the one at $f(j)=f_{0}$ will be zero (Figure $6 \mathrm{~d}$ ). This is the special class of : 
waveforms referred to earlier for which the DFT and the continuous Fourier transform are the same within the scaling constant, $\Delta t$. The more general case is where $n_{0}$ is not an integer. Then $f_{0}$ falls between two of the discrete frequencies and all of the DFT coefficients are nonzero (Figure. 6e). These additional frequency components are çalled "leakage" and are inherent in the DFT because of the required time-domain truncation. Recalling that use of the DFT implies a periodic function in the time-domain, the effect of truncating the signal at other than an integer multiple of its period is to create a periodic function. with discontinuities; as shown in Figure:6e. Physicá1ly, it would be expected that these discontinuities would introduce additional frèquency components.

$\because$ There is no way to avoid leakage, but its effect can be minimized if the data are multiplied by an appropriate nonrectangular time-domain truncation function, called a data window (Figure 2, block 6). A suitable data window will eliminate the discontinuities at each end of the data record and will have a transform with smaller sidelobes than those of the rectangular window. Spectra calculated from data with a rectangular window are called raw spectra, while those calculated from data truncated with a window which has less leakage are called modified spectra. A data: window which the authors have found to be useful with nonrandom data is called; equivalently, the Hanning, 17 cosine-bel1, ${ }^{17}$ or sine-squared 15 window. This window function is : given by

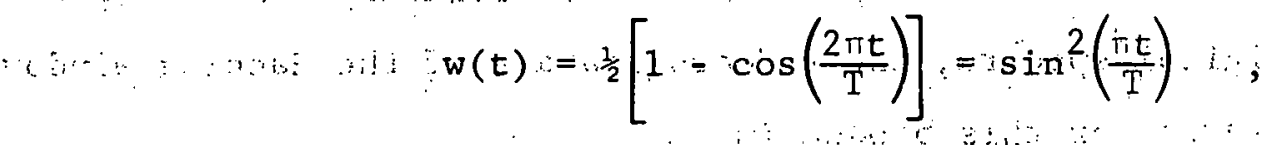

where $T$ is the truncation interval: This window and its transform are compared to the rectangular window and its transform in Figure 7 . The magnitude of the DFT coefficients for the Hanning window have been multiplied by 2.0 (ratio of window areas in the time-domain) so that the $f=0$ coefficient "will match that for the rectangular window. The sidelobes" of the Hanning window have much smaller magnitude than those of the rectangular 


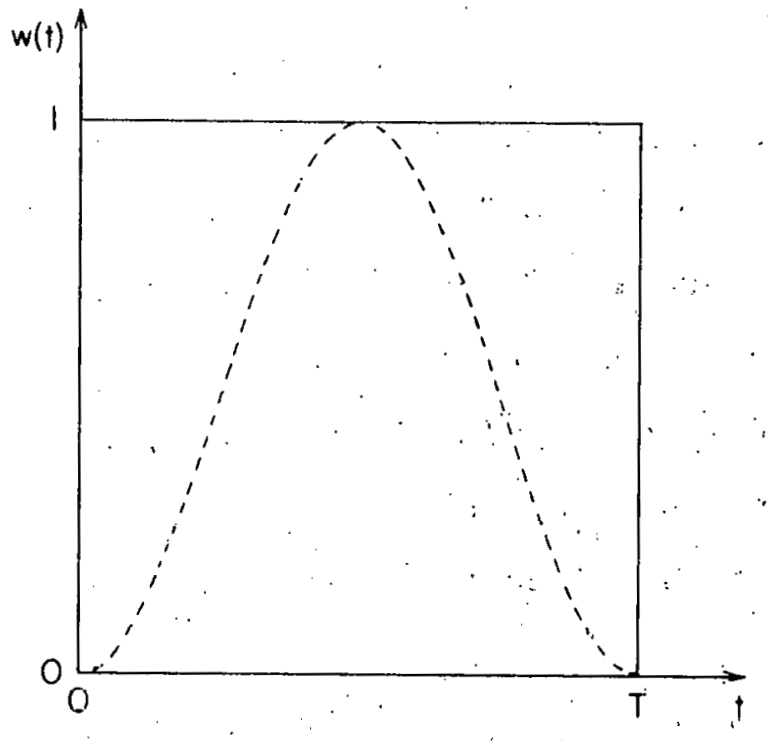

a: Window

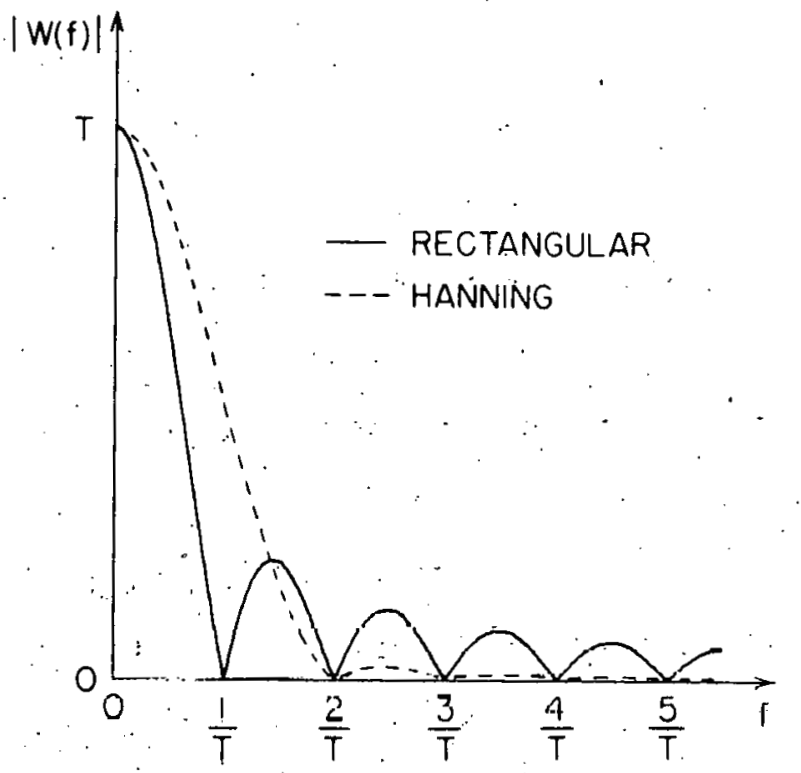

b. Transform

Figure 7. Data windows

window, demonstrating the șmaller leakage associated.with this windnw. Quantitatively, the peak sidelobe magnitude of the Hanning window is propurliullal to $\left|f_{\odot}-f\right|^{-3}$ compared to $\left|f_{0}-f\right|^{-1}$ for the rectangular window. Unfortunately, the width of the mainlobe of the transform is doubled. Thio is a consequence of the Fourier transform property that compression of a time-domain function results in expansion of the frcqucncy-domain trausfurm. In calculating digital spectra, the reduced lẹakage of the Hanning window more than compensates for this broadening.

Fnr sampled data, the llannjug wirluw function; w(k), is given by

$$
w(k)=\frac{i}{2}\left[1-\cos \left(\frac{2 \pi k}{N}\right)\right]=\sin ^{2}\left(\frac{\pi k}{N}\right),
$$

where $k=0,1, \ldots, N-1$. The effectiveness of the Hanning window in reducing leakage is shown in Figure 8 . The time function, $g_{c}(t)=\cos (2 \pi f t)$ 


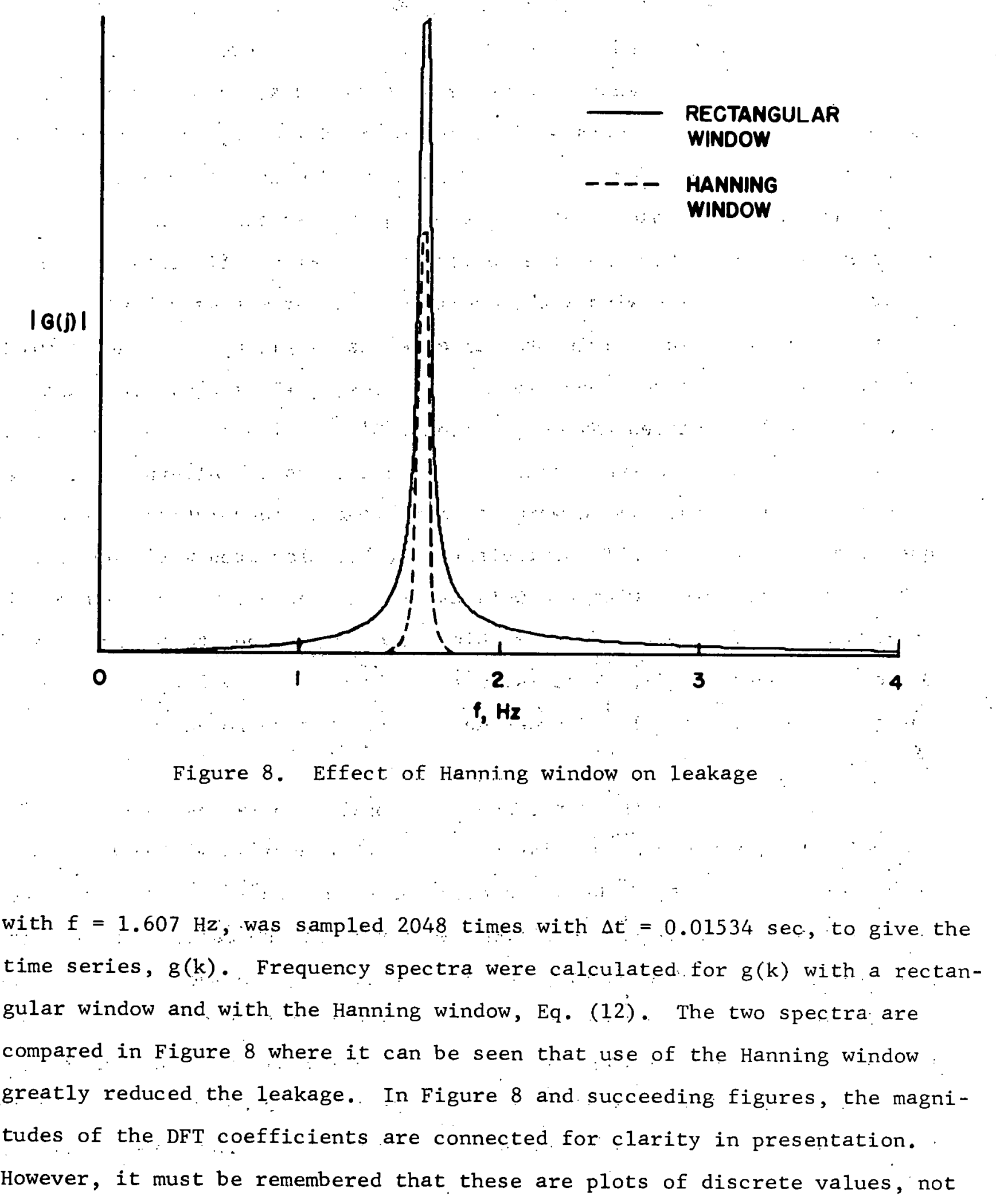


continuous functions. Another window which is frequently suggested is the ten-percent cosine-be11. 17 With this window, the left and right halves of a cosine bell are applied to the first and last ten percent of the data, respectively, and the central 80 percent of the data are not modified. Although this window is apparently good for random data, the authors found it to be definitely inferior to the Hanning window for nonrandom data. It should be noted that the same modified spectrum can be obtained by operations on either the time series or the DFT coefficients. In particular, tor the Hanning window the modified spectrum may be calculated as described above by multiplying the time series, $g(k)$, by the Hanning winduw function, $w(k)$, to give a modified time series, $g^{\prime}(k)=g(k) \cdot w(k)$, and then calculating the modified DFT coefficients; $G^{\prime}(j)$, with $g^{\prime}\left(k_{6}\right)$. The proccdure outlined in Figure 2 assumes that this method is used. Alternately; sinre multiplication in the time domain is equivalent to convolution in the frequency domain, the raw DFT coefficients, $G(j)$, calculated with the series $g(k)$, can be convolved with the weights, $-\frac{1}{4}, \frac{1}{2}$, and $-\frac{1}{4}$, which are the only nonzero DFT coefficients of whe Hanning window function, to give modified DFT cuefficlents, $G^{\prime}(j)$. That is, $G^{\prime}(j)=\frac{1}{4}[-G(j-1)+2 G(j)-G(j+1)]$, for $j=0,1, \ldots, N-1$. Furthei details on this approach arc given in Ref. 15 and 17 .

Atter windowing the data, one additional step may be required before the DFT coefficients are calculated. Many FFT programs require that the number of data points, $N$, be an integral power of two (i.e., $N=2^{n}$ ) since the FFT computation is most efficient when this is the case. Mixed-radix piugrams which àccept arbitrary values of $\mathrm{N}$ may require much more computer storage when $\mathrm{N}$ is not a power of two and the computation time can be excessive if $\mathrm{N}$ is not highly composite, that is, factorable into several small factors. For instance, relative computation times on a CDC 6600, using subroutine FOURT with a set of real data, are given below for three values of $\mathrm{N}$ : 


\begin{tabular}{rcr}
$\frac{N}{N}$ & Factorization & \multicolumn{2}{c}{$\begin{array}{c}\text { Relative Ti } \\
\text { DFT Comput }\end{array}$} \\
4096 & $2^{12}$ & 1.0 \\
4097 & $17 \times 241$ & 38.0 \\
4099 & prime & 603.5
\end{tabular}

Therefore, it is advantageous to have $\mathrm{N}$ be a power of two. A standard procedure is to extend the data to the next integral power of two by adding zeros at the end of the data set (Figure 2, block 7). If a mixedradix program is available, the data can be extended with zeros to the next highly composite number. A table of numbers containing no prime factor greater than five is provided in Ref. 13 and is useful in selecting efficient values of $\mathrm{N}$ for mixed-radix programs.

The frequency resolution of the DFT is $\Delta f=1 / N \Delta t$. Thus, it appears that adding zeros to increase $\mathrm{N}$ is a means of increasing the resolution. However, the leakage prevents this from being a true increase in frequency resolution. The half-width of the mainlobe of the window transform is $\Delta \mathrm{f}=1 / \mathrm{T}$. for a rectangular window or $2 / \mathrm{T}$ for a Hanning window where $\mathrm{T}$ is the total time span of the actual data. Adding zeros does not increase $\mathrm{T}$ and, therefore, does not improve the DFT resolution.

While the addition of zeros to the data series does not change the DFT magnitude for a given frequency [i.e., for a given value of $j / N$, the summation in Eq. (3) remains the same when zeros are added to the series, $\mathrm{g}(\mathrm{k})]$, it does shift the frequencies at which the coefficients are calculated, thereby changing the appearance of the spectrum. If the data are not extended with zeros, the leakage appears smooth (as in Figure 8) because there is only one DFT coefficient per sidelobe and the location of the coefficient is the same relative to each sidelobe. However, when zeros are added, the coefficients are no longer in phase with the sidelobes and, depending on the number of zeros added, the spectrum can be either smooth or very ragged. If enough zeros are added, say five or more zeros per data 
sample, the sidelobes of the window spectrum will be clearly defined and the spectrum of the data may appear similar to that shown in Figure 6c. Such spectra are generally misleading since they emphasize the spectrum of the window at the expense of the data spectrum. Raw and modified (Hanning window) spectra calculated for data sets which were extended with zeros are presented in Figure 9. The time function, $g_{c}(t)=0.25 \cos \left(2 \pi f_{1} t\right)+$. $\cos \left(2 \pi f_{2} t\right)$, where $f_{1}=0.493 \mathrm{~Hz}$ and $f_{2}=1.607 \mathrm{~Hz}$, was sampled $\mathrm{N}$ times with $\Delta t=0.01534 \mathrm{sec}$ for these examples. In all cases, 2048 values were used in computing the DFT coefficients. Thus, the number of added zeros in each case is 2048 - N. In Figures $9 \mathrm{a}$ and $9 \mathrm{~b}$ the raw and modified spectra are presented for $N=2048$, i.e., no zeros have been added to the data set. The spectra have leakage similar to that shown in Figure 8 . The spectra in Figures 9c and 9d are for 1707 samples of data extended with 341 zeros. The raw spectrum is very rough and could lead the user to believe that there are extraneous frequencies present in the data. This roughness is a result of the sidelobes of the window spectrum. In this example, the DFT resolution is $0.0318 \mathrm{~Hz}$ and the sidelobes have a width of $0.0382 \mathrm{~Hz}$. Therefore, each of the minor "pcaks" consists of points on several adjoining sidelobes and does not define a single sidelobe. 'l'he modified spectrum is not significantly changed by the addition of zeros. In Figures $9 e$ and $9 f$ spectra are presented for 1365 samples extended with 683 zeros, and the results are similar to those of rigures $9 c$ and $9 d$. From these. figures it can be seen that the use of a modjified spectrum is almost.essential if the data have been extended with zeros.

In some cases it nay be advantagevus to truncale lle dala selies instead of extending it. This is obviously the case if the number of data samples is only slightly greater than a power of two. If the data are to be truncated, this operation must be performed before the bias is removed and the data window applied, that is, at step 4 of the procedures outlined in Figure 2 . 


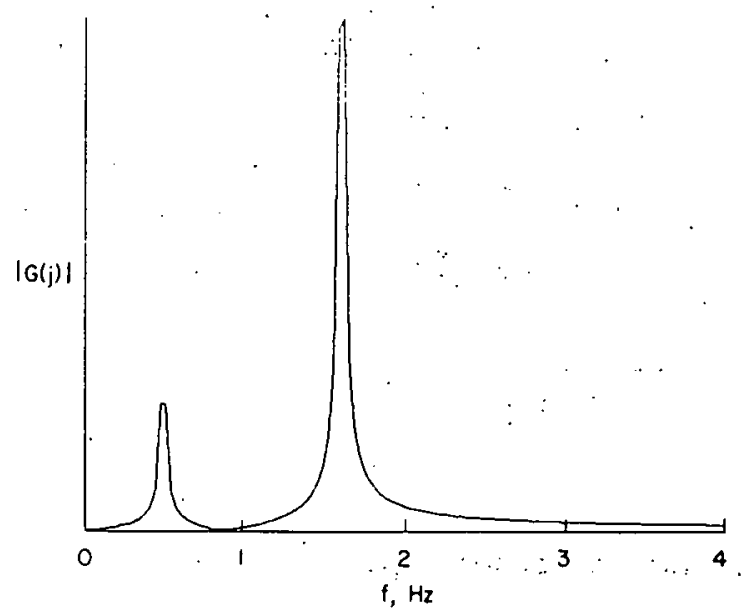

a. Raw spectrum, $\mathrm{N}=2048^{\circ}$

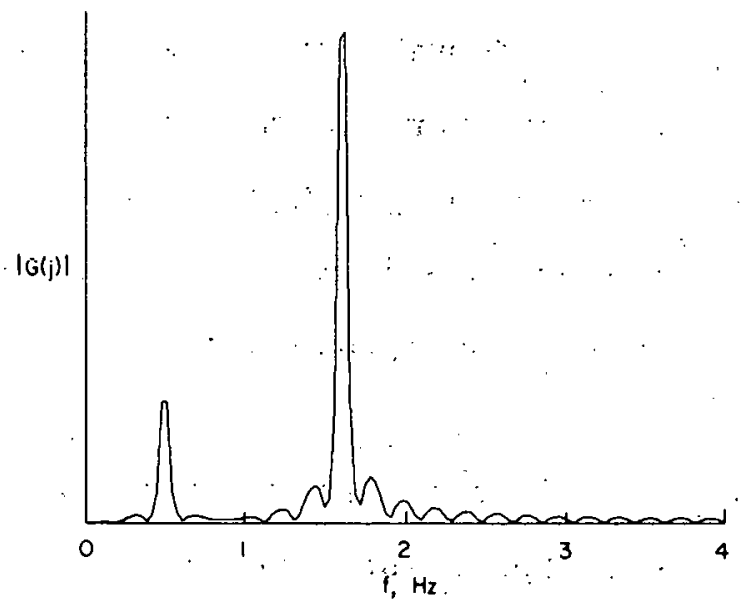

c. Raw spectrum, $\mathrm{N}=1707$

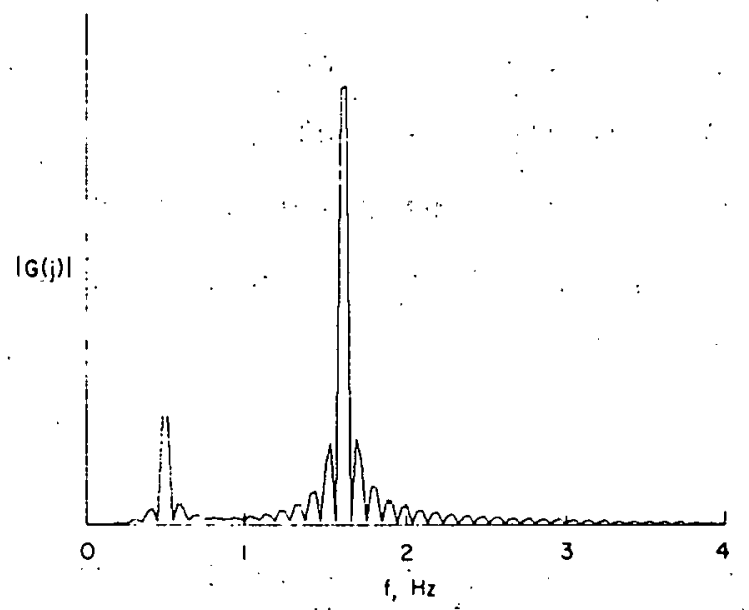

e. Raw spectrum; $N=1365$

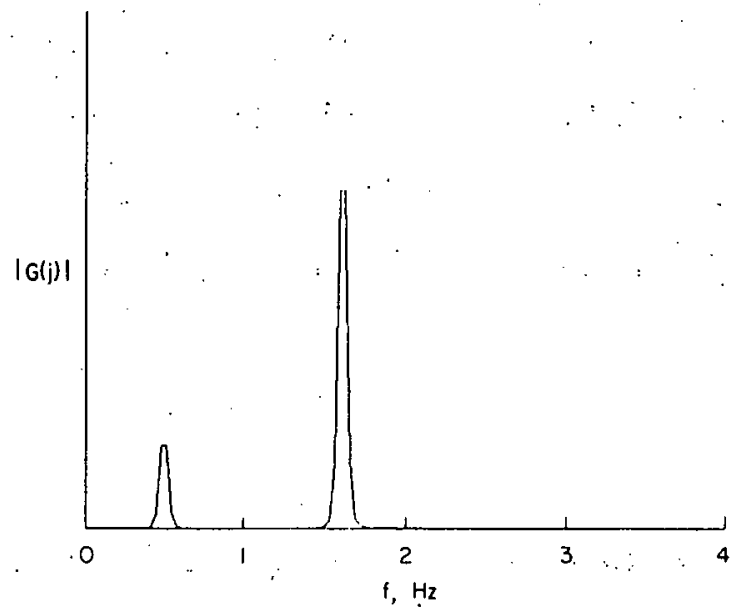

b. Modified spectrum, $N=2048$

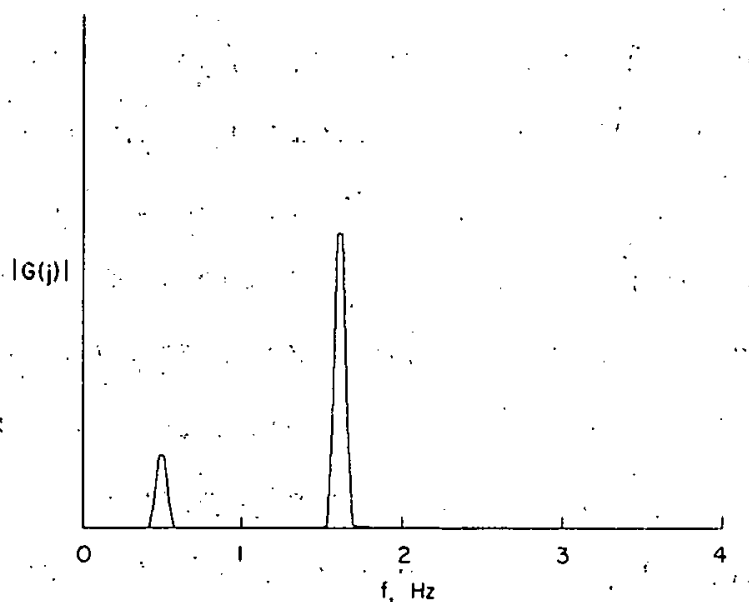

d." Modified spectrum, $N=1707$

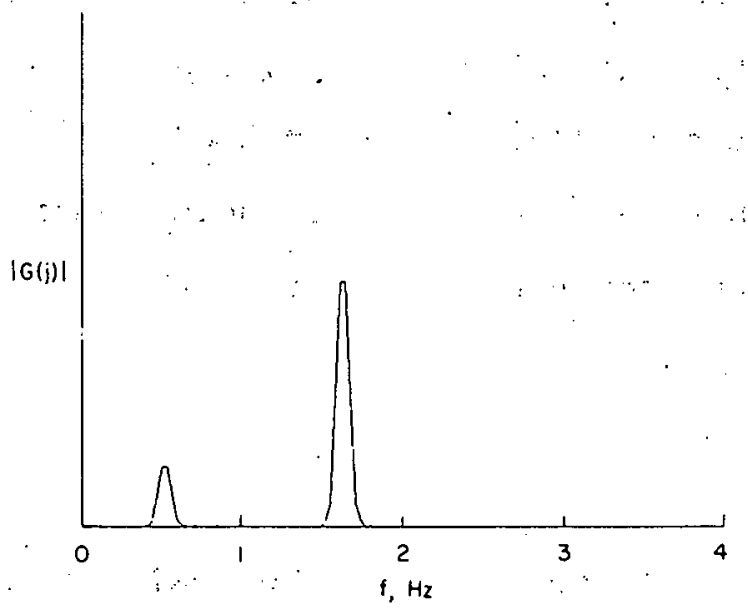

f. Modified spectrum, $N=1365$ Figure 9. Spectra of data sets extended with 2048 - N zerós 
After the data are windowed and extended with zeros (if necessary), the complex DFT coefficients are calculated using the FFT algorithm (Figure 2 , block 8 ). The real and imaginary parts of the coefficients are combined to obtain the magnitude of the coefficients,

$$
|G(j)|=\sqrt{[\operatorname{Re} G(j)]^{2}+[\operatorname{ImG}(j)]^{2}},
$$

where $\operatorname{Re} G(j)$ and $\operatorname{Im} G(j)$ are the real and imaginary parts of $G(j) . *$ For real time-domain data, the frequencies corresponding to the DFT coefficients are $f(j)=j / N \Delta t$, where $j=0$ to $N / 2$, giving a frequency range of zero to

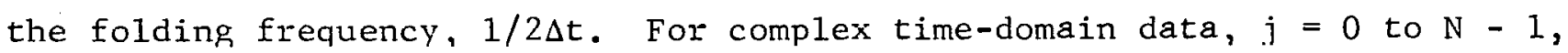
where the DFT coefficients for $j=0$ to $N / 2$ correspond to the positive frequencies, $f(j)=j / N \Delta t$, and the DFT coefficients for $j=N / 2+1$ to $N-1$ correspond to the negative frequencies, $f(j)=-(N-j) / N \Delta t$. The frequency range is $-1 / 2 \Delta t$ to $1 / 2 \Delta t$, and the transform does not possess conjugate symmetry as is the case with real data.

The relationship between the DFT coefficients and the spectral estimate depends upon the type of data analyzed. For noise-1ike. (random) data, it is conventional to provide an estimate of the power spectrum of the original signal by plotting the square of the magnitude of the set of complex DFT coefficients, $|G(j)|^{2}$, as a function of frequency. The power spectral density function, with $2|G(j)|^{2} / T$ as the dependent variable, is also used. These are appropriate choices for aerodynamic studies relating to turbulence, . unsteady pressures, etc. However, for nonrandom data, the magnitude of the

*Inspection of the real and imaginary parts of the cocfficicnts is not generally of much value since a constant shift in the time domain results in a phase shift in the frequency.domain. Thus, shifting the starting point of the time series by as little as one sample can drastically alter the appearance of the real and.imaginary parts of $G(j)$. The magnitude, $|G(j)|$, is not changed by a time shift. 
DFT coefficients is proportional to the amplitude of the signal components and an amplitude'spectrum, with $|G(j)|$ plotted as a function of frequency, is more appropriate. In fact, if the data are not noisy, use of the power spectrum can result in a significant loss. of information since sma11magnitude coefficients will be suppressed. This is demonstrated in a later section of this report. The coefficients should always be plotted (Figure 2, block 9) for the full frequency range (0 to $1 / 2 \Delta t$ for real data or $-1 / 2 \Delta t$ to $1 / 2 \Delta t$ for complex data) to permit detection of significant frequencies. Expanded plots for smaller frequency ranges may then be used to examine details of the spectrum.

Regardless of the type of spectra used, an important fact must be remembered-digital spectra are discrete, not continuous: Therefore, the magnitudes of the DFT coefficients may not correspond to the peak magnitudes of the continuous transform. This is especially true in the analysis of nonrandom data. For example, if the signal frequency $f_{0}$ is halfway between two consecutive discrete frequençies, the two corresponding DFT coefficients will have magnitudes equal to $2 / \pi(\approx 0.64)$ times the continuous transform magnitude for $f=f_{0}$ if a rectangular window is used. Other types of windows introduce different scaling factors. Furthermore, leakage from other frequency components contributes to the uncertainty in the spectrum amplitude at a particular frequency. Finally, even for a continuous Fourier transform, the magnitude of the spectrum can be dependent upon factors other than the signal amplitude. For example, the spectrum of a damped cosine wave is nonzero at all frequenciès, and has a peak value which is proportional to the initial amplitude of the wave divided by the damping factor. Hence, digital spectra should be used only to identify waveform component frequencies, not to quantify the amplitudes of the components.

A basic limitation of digital spectra is their inability to accurately resolve the frequencies present in a signal for which only a few cycles of data are available. The ratio of the DFT resolution, $\Delta f$, to the 
signal frequency, $f_{0}$, is a measure of the relative accuracy with which the trequency can be determined. From Eqs. (6) and (11),

$$
\frac{\Delta f}{f_{0}}=\frac{1}{N \Delta t} \cdot \frac{T}{n_{0}} \text {, }
$$

where $n_{0}$ is the number of cycles of data available for analysis. For data with no added zeros, $\mathrm{T}=\mathrm{N} \Delta \mathrm{t}$ and

$$
\frac{\Delta f}{f_{0}}=\frac{1}{n_{0}}
$$

When the data are extended with zeros, the resolution is equal to the mainlobe half-width, $1 / \mathrm{T}$, and Eq. (14) again applies. Therefore, it is obvious that the frequency cannot be accurately. determined from a limited number of cycles of data.

Random (Gaussian) noise in the data is not generally a problem since its frequency content will be spread uniformly over the entire-spectrum. If noise appears to be a problem in a specific application, its influence on the spectrum can be reduced by using $|G(j)|^{2}$ or $\log |G(j)|$ as the dependent variable. If long data records are available, the data can be broken into sections (possibly overlapping), a spectrum calculated for cach scction, and tho cet of cpcctra avcragcd. 18 Thio tcchniquc is cffective in redueing the effects of even extreme noise. ${ }^{19}$

It has been assumed in this discussion that the data are stationary, that 1s, the frequency content is not varying with time. The spectrum ot a set of nonstationary:data will have the frequencies "smeared". The smearing can be reduced by breaking the data into smailer sections which are analyzed separately. 
Digital filters provide a computational means of attenuating undesired frequencies in a set of time-dependent data. Thus, they are analogous to continuous electronic filters. However, digital filters are much more versatile than electronic filters and can be easily and precisely tailored to each application. These advantages are especially apparent at the low frequencies $(0.1$ to $10.0 \mathrm{~Hz}$ ) which occur in dynamic data. The theory and design of digital filters has been thoroughly. covered in the literature. 20

A major factor in the selection of a digital filter is whether the data are to be real-time or nonreal-time processed. If rea1-time processing is required, the filter algorithm must be fast enough so all computations: can be performed between samples. For nonreal-time data processing, all of the data must be stored and, hence, the quantity of data to be analyzed must be limited. Dynamic data from ground test facilities can usually be processed in nonreal time.

There are two basic types of digital filters, recursive and nonrecursive. A nonrecursive filter is a filter for which each output sample is determined as a weighted sum of the input samples. A recursive filter is a filter for which each output sample is determined as a weighted sum of the input samples and the past output samples. A general expression for both types of filters is

$$
\begin{gathered}
\bar{g}(k)=\sum_{m=-n}^{n} a_{m} g(k-m)-\sum_{m=1}^{n} b_{m} \bar{g}(k-m) ; \\
k=0,1, \ldots, N-1 \ldots,
\end{gathered}
$$

where $\mathrm{k}$ is the index of the present sample, $\mathrm{g}(\mathrm{k})$ is the input to the filter at $t=k \Delta t, \bar{g}(k)$ is the output of the filter at $t=k \Delta t, a_{m}$ and $b_{m}$ are 
weights which determine the type, of filter, and $n$ is the order of the filter. If at least one $a_{m}$ and $b_{m}$ are nonzero, the filter is recursive. If all the $\mathrm{b}_{\mathrm{m}}$ are zero, the filter is nonrecursive. In general, nonrecursive filters are easier to design than recursive filters. However, nonrecursive filters tend to require a larger number of terms (i.e., a higher order) than a recursive filter in order to obtain a particular rate of frequency attenuation. Because recursive filters have feedback, truncation or round-off errors may cause them to become unstable, resulting in an oscillatory output. Nonrecursive filters are inherently stable.

Both types of filters have a time delay between input and output samples when only past and present samples are used, as in real-time data processing. Nonrecursive filters can be designed to have a linear phase shift (i.e., constant time delay at all frequencies), whereas recursive filters can. only approximate linear phase. Thus, if several frequencies are present in the signal; use of a recursive filter will result in waveform distortion. A zero-phase-shift filter is useful when the input and output data are to be compared, since there is no time delay between input and output samples with this type of filter. 'Such filters are realizable only for nonreal-time processing. A nonrecursive filter will have zero phase shift if both pact and future samplcs arc uscd with symmctrical wcights (i.c., $a_{-m}-a_{m}$ ) to conipute the piesent vutput value. Tu ullaiı celu pliase shift with a recursive filter, the data are passed through the filter twice, once with the data reversed. ?1

Filters can be implemented by use of Eq. (15) or by means of Fourier transforms. A simple, nonrecursive filter of the latter type will be used to illustrate how a digital filter functions. For a given set of timedependent data, the DFT coefficients are calculated, thereby determining the frequency content of the data set. The frequency range of these coefficients is zero to $\mathrm{f}_{\mathrm{S}} / 2$. Next, all coefficients corresponding to frequencies greater than the cutoff frequency $f_{C}\left(<f_{S} / 2\right)$ are set equal to zero. This step is equivalent to applying a rectangular window in the frequency domain. Finally, 
the inverse DFT is used to calculate a filtered time series from the truncated DFT coefficients. It appears that these operations will result in an ideal filter, that is, a filter which has unit gain for $0 \varsigma \mathrm{f}=\mathrm{f}_{\mathrm{C}}$ and zero gain for $f>f_{C}$. However, leakage, which is always present in the DFT coefficients, prevents realization of this ideal filter. Sidelobes from frequencies below $f_{C}$ will be lost while sidelobes from frequencies, above $f_{C}$ will be retained. Furthermore, the discontinuity at $f=f_{C}$ in the frequency domain results in oscillations in the filter gain known as Gibbs phenomenon. If the frequency-domain window is modified to have a continuous rolloff, for example, by use of a cosine bell. (similar in shape to the right half of the Hanning window, Figure 7a), this transform technique can provide a satisfactory digital filter.

The digital filter which was used in the applications presented in this report is known as the Martin-Graham filter. 22,23,24 It is nonrecursive and is designed for nonreal-time data processing. The gain characteristics of the filter can be accurately specified and easily changed. The filter has zero phase shift, a characteristic which is necessary if the input and output data are to be compared. The usual filtering operations-low-pass, high-pass, band-pass, and band-reject-can be performed with this filter. The basic low-pass filter, from which the other filter operations are derived, has a gain characteristic distinguished by a cosine-bell frequency rolloff. The filter gain, $H(f)$, is defined by

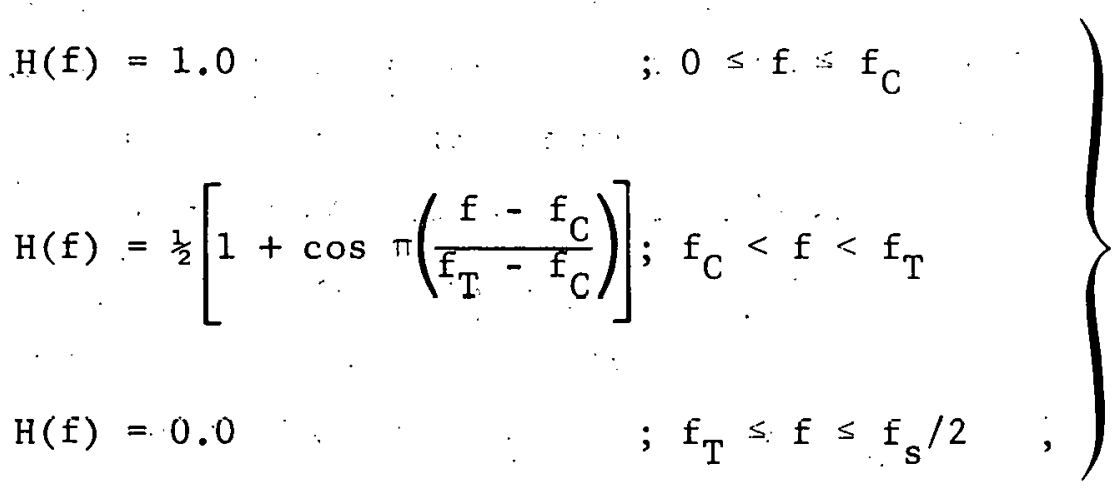


where $f_{C}$ is called the cutoff frequency and $f_{T}$ the termination frequency. The three frequency intervals given in Eq. (16) are referred to as the passband, transition band, and stopband, respectively. For this filter, the gain is 1.0 at the cutoff frequency. However, the gain characteristics of digital filters are frequently defined, similar to electronic filters, with. the cutoff frequency corresponding to the $-3 \mathrm{db}$ or half-power point, that is, the gain of the filter is approximately 0.71 at the cutoff frequency. This difference in terminology is mentioned as a caution to the user. From Eq. (16) it is seen that $H(f)$ and its first derivative, $d H / d f$, are continuous. Therefore, the Martin-Graham filter will have much smaller oscillations than the ideal (rectangular) filter described earlier.

The filter is implemented by means of the nonrecursive equation 「see. Eq. (15)],

$$
\bar{g}(k)=\sum_{m=-N_{w}}^{N_{w}} h(m) g(k-m) ; k=0,1, \ldots, N-1,
$$

where $g(k)$ is the input value at $t=k \Delta t, \bar{g}(k)$ is the output (filtered) value at $t=k \Delta t$, and $h(m)$ are the $2 \mathrm{~N}_{w}+i$ filter weights. Note in Eq. (17) that the present filtered value is determined from past, present, and future data samples. By using future samples $(m<0)$, the filter will have zero phase shift but is obviously limited to nonreal-time applications. An expression for the filter weights, $h(m)$, is derived in Ref. 22 by calculating the continuous inverse Fourier transform of the filter gain function, $\dot{H}(f)$. Equation (17) represents a discrete convolution of the weights and input data. This operation is analogous to calculating the direct Fourier transform of the data, multiplying this transform by the filter gain function, and,calculating the inverse transform of the product.

Since the filter weights are derived with a continuous inverse transform, an infinite number of weights would have to be used with Eq. (17) to 
provide exactly the filter gain, $H(f)$, defined in Eq. (16). Equations are given in Ref. 22 for calculating the actual gain of the digital filter for a finite number of weights, $\mathrm{N}_{\mathrm{w}}$. Determination of the necessary number of weights is a trial-and-error process. It was recommended in Ref. 23 that, for the maximum difference between $H(f)$ and the actual gain to be approximately one percent, the number of weights to be used should be

$$
\mathrm{N}_{\mathrm{w}} \geq \frac{1.25}{\Delta \mathrm{t}\left(\mathrm{f}_{\mathrm{T}}-\mathrm{f}_{\mathrm{C}}\right)}
$$

Writing this equation in terms of the folding frequency, $f_{s} / 2$, gives

$$
\mathrm{N}_{\mathrm{w}} \geq \frac{2.5}{\left(\mathrm{f}_{\mathrm{T}}-\mathrm{f}_{\mathrm{C}}\right) /\left(\mathrm{f}_{\mathrm{s}} / 2\right)}
$$

Thus, if $f_{T}-f_{C}$ is 25 percent of the folding frequency, a filter with $\mathrm{N}_{\mathrm{w}}=10$ would give approximately one percent accuracy. If $f_{\mathrm{T}}-f_{\mathrm{C}}$ is reduced to 2.5 percent of $\mathrm{f}_{\mathrm{s}} / 2$, a filter with $\mathrm{N}_{\mathrm{w}}=100$ would be required. To mini-. mize computation time, the transition band, $\mathrm{f}_{\mathrm{T}}-\mathrm{f}_{\mathrm{C}}$, should be as large as the application will permit.

Data which are less than $\mathrm{N}_{\mathrm{w}}$ samples from either end of the data set cannot be filtered since the $\mathrm{N}_{\mathrm{w}}$ weights must be applied symmetrically [see Eq. (17)]. If the data set is large, the first and last $\mathrm{N}_{\mathrm{w}}$ points may be discarded in the filtering process. However, if these points are to be retained, the data set must be extended at each end by adding $\mathrm{N}_{\mathrm{w}}$ artificial samples. In Ref. 22 it is recommended that the artificial data be a mirror image of the first (and last) $\mathrm{N}_{\mathrm{w}}$ data samples. In the present applications, the artificial data were held at a constant value, equal to the first or last data sample. Since both of these techniques will usually result in a slope discontinuity at the beginning and end of the actual data, some distortion will be present in the first and last $\mathrm{N}_{\mathrm{w}}$ filtered values. 


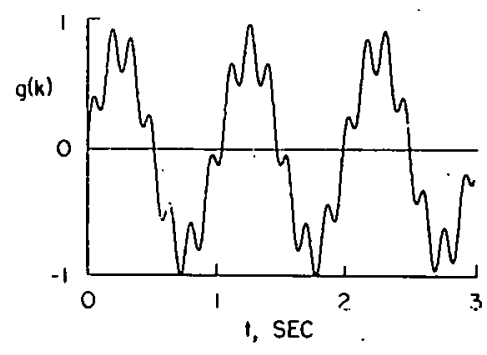

unfiltered data

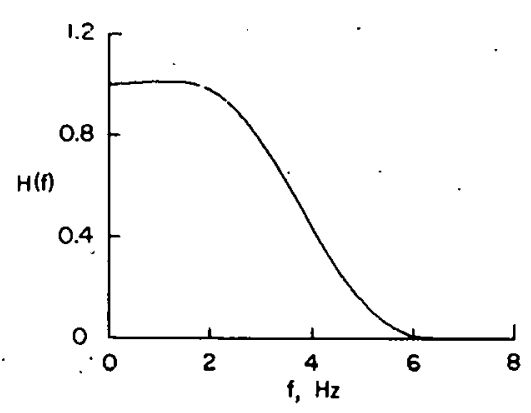

filter gain

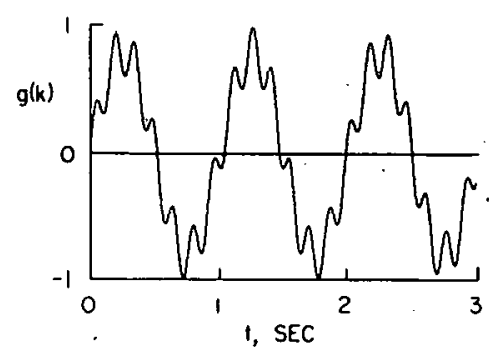

unfiltered data

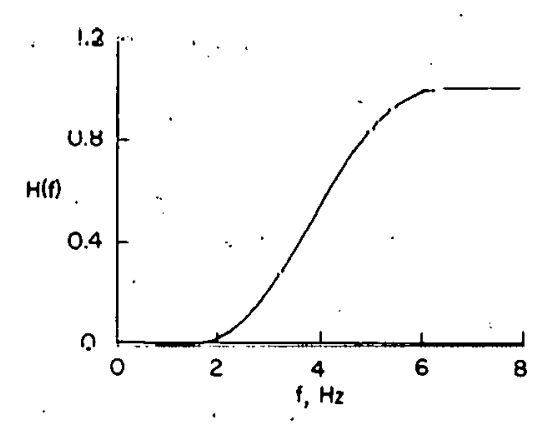

filter gain

filter gain

b. High-pass filter

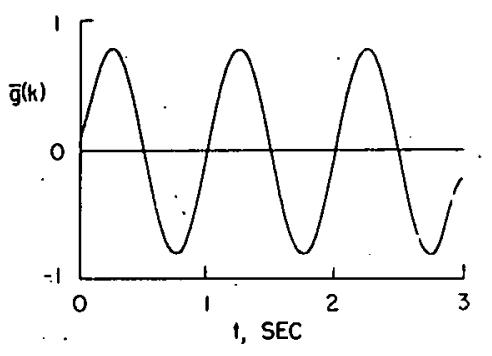

filtered data

\section{a. Low-pass filler}

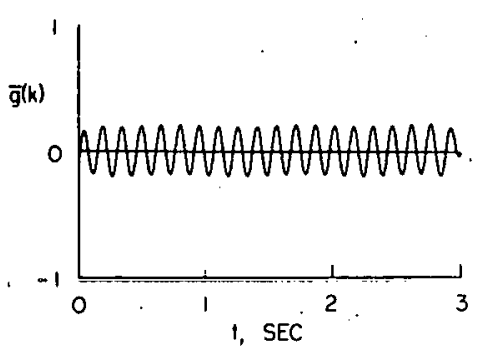

filtered data

Figure 10. Sample results for the Martin-Graham filter

Sample results for the Martin-Graham filter are presented in Figure 10. The unfiltered data were generated with the function, $g_{c}(t)=0.8 \sin \left(2 \pi f_{1} t\right)+0.2 \sin \left(2 \pi f_{2} t\right)$, where $f_{1}=1,0 \mathrm{~Hz}$ and $f_{2}=6.6 \mathrm{~Hz}$. A time interval of $0.004 \mathrm{sec}$ was used, and 750 samples were generated. The low-pass filter cutoff frequency, $f_{C}$, was $1.5 \mathrm{~Hz}$, and the termination frequency, $\mathrm{f}_{\mathrm{T}}$, was $6.1 \mathrm{~Hz}$. Equation (18) indicates that at least 68 filter weights are required for approximately one-percent accuracy. In the example, 75 weights were used and the gain was accurate to 1.1 percent. As seen in Figure 10a, the low-pass filter passed only the low-frequency $(1.0 \mathrm{~Hz})$ component and, in Figure 10b, the high-pass filter passed only the high-frequency $(6.6 \mathrm{~Hz})$ component. A small amount of distortion can be seen in the first 
$0.3 \mathrm{sec}\left(=\mathrm{N}_{\mathrm{w}} \Delta t\right)$ of the filtered data as a result of the artificial data used to start the filtering operation.

Before filtering sampled data (Figure 2, block 10), it is advisable to first calculate the digital spectrum so that the frequency content of the data is accurately known. "Because digital filters operate on sampled data, aliasing errors will occur if the sampling theorem is violated. As was the case with the transform of a sampled signal (Figure 5), the filter response will be replicated at intervals, $f_{s}=1 / \Delta t$ (Figure 11$)$. Two types of aliasing error may occur. First, if the data are not bandlimited to $0 \leq \mathrm{f}^{\prime}<\mathrm{f}_{\mathrm{s}} / 2$ (e.g., by electronic prefiltering), any frequencies in the

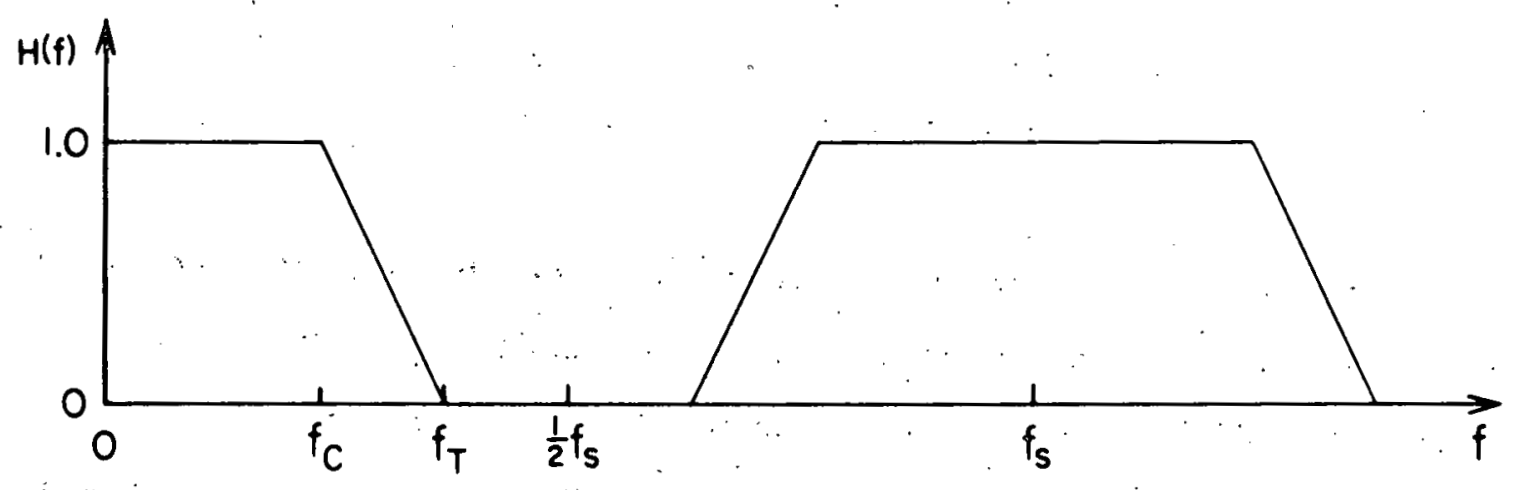

a. Filter gain function for $\mathrm{f}_{\mathrm{s}}>2 \mathrm{f}_{\mathrm{T}}$

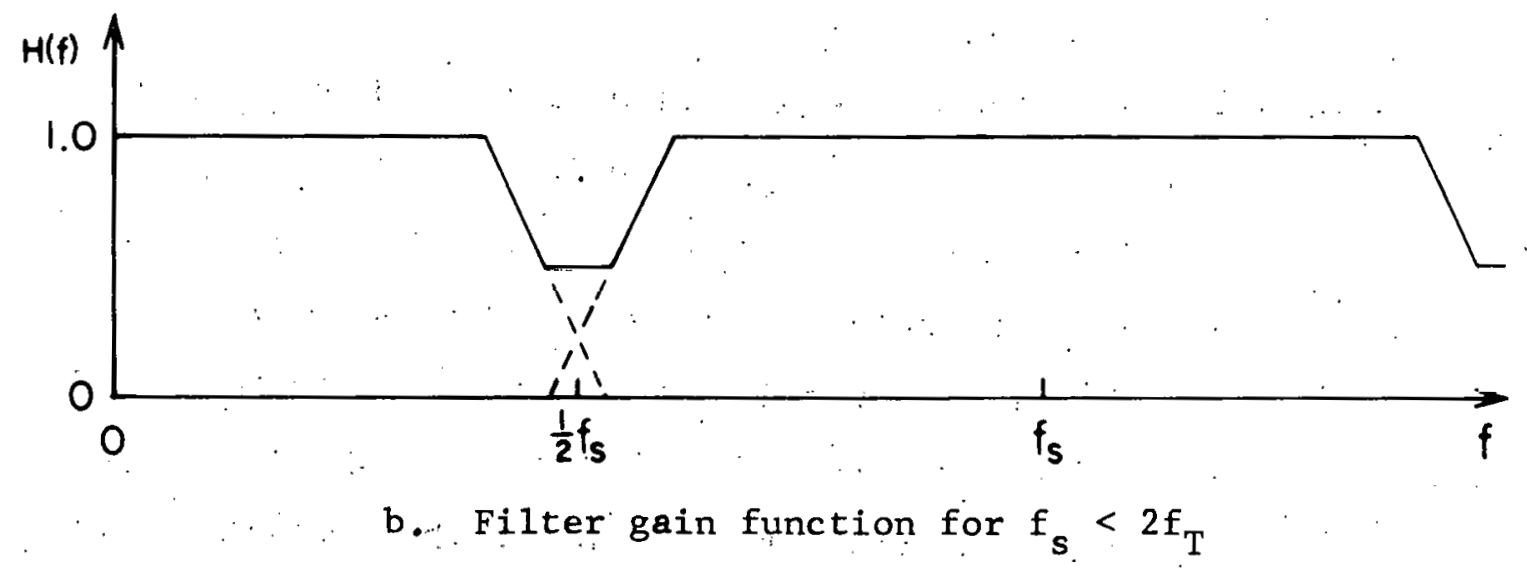

Figure 11. Aliasing error in digital filters 
intervals $\mathrm{nf}_{\mathrm{S}}-\mathrm{f}_{\mathrm{T}}$ to $\mathrm{nf}_{\mathrm{S}}+\mathrm{f}_{\mathrm{T}}$, where $\mathrm{n}$ is any integer, will be passed by the filter (Figure'11a). Second, if the termination frequency, $\mathrm{f}_{\mathrm{T}}$, is greater than $f_{s} / 2$, the filter gain will be incorrect because the replicated gain curves are additive (Figure 11b).

After the data are digitally filtered, two optional checks on the results may be desirable (Figure 2, blocks 11 and 12). First, if a zerophase-shift filter were used, the residuals (i.e., the difference between filtered and unfiltered data) may be plotted to permit examination of discarded frequency components. An advantage of digital filters is that the residual can be easily obtained, whereas it is very difficult to obtain residuals with an electronic filter. Second, the digital spectrum of the filtered data may be calculated to demonstrate that the filtering operation modified the data as the user intended.

\section{Verification of Procedures}

Although digital trequency techniques can be of considerable value. in the analysis of dynamic data, there are pitfa11s which can lead to erromeous results or incorrect interpretation of results. While some of the errors may be obvious, others are not. It is recommended that each, step in the analysis be carefully checked. Some suggested procedures are listed. below.

Sampling: The output of a low-distortion oscillator, with frequency determined from an electronic counter, is recorded with the analog rccordcr. Several. frequeicies, bimulaling those present in the actual data, should be recorded. The simulated dața are then prefiltered, sampled, and plotted to check the sampling process. Calculating the digital spectra of the simulated data will show if undesirable frequency components (e.g., $60 \mathrm{~Hz}$ noise) are appearing during recording and sampling: 
Digital Spectra: The best way to check spectral calculations, and to gain a better understanding of digital spectra, is through the analysis of computer-generated data. With generated data, the effects of nonperiodicity, windows, added zeros, and multiple frequency components can be easily determined. The data-generation program should have the capability of providing single and multicomponent sine waves with amplitude, frequency, phase angle, and damping factor as variables. Different definitions of the DFT exist, scaling factors are required to approximate the continuous Fourier transform with the DFT, and FFT codes use various schemes to store the transform results. Therefore, it is recommended that the first use of a new FFT code should be to transform a function which has a known continuous transform. A good example is the function shown in Figure $1, g_{C}(t)=e^{-t}$, which has the transform, $G_{C}(f)=(1-i 2 \pi f) /\left[1+(2 \pi f)^{2}\right]$. If $g_{c}$ is sampled with $N=512$ and $\Delta t=0.0156 \mathrm{sec}$, the continuous and discrete transforms should agree within 0.3 percent for $f \leq 2 \mathrm{~Hz}$ (the sampled function should be equal to $g_{c} / 2$ at $t=0$ because of the discontinuity). This type of calculation will show if the code is being used correctly.

Digital Filters: Again, a program that permits the filter to be checked with computer-generated data is usetul for verffylub filter gain characteristics and determining filter time lag. An interesting way to verify gain characteristics is as follows. Generate a unit area impulse function by giving one data sample, near the middle of the series, a value of $1 / \Delta t$ and setting ald other samples to zero (the value of $\Delta t$ is determined by the desired folding frequency, and the number of samples is determined by the desired frequency resolution of the spectrum and must be greater than $2 \mathrm{~N}_{\mathrm{w}}$ for evaluation of the Martin-Graham filter). The digital spectrum of this impulse function has unit 


\begin{abstract}
magnitude for all frequencies from zero to the folding frequency. Filter the generated series and calculate the digital.spectrum of the filtered series. The spectrum will display the discrete gain characteristics of the filter.
\end{abstract}

\title{
Selected Applications
}

Threp aerodynamic testing applications have been selected to demonslrate digital spertral annlygie and filtering. Allluugh these applications use actual or simulated wind tunnel data, the analysis methods can be applled to ballistic range or flight data if sufficient data are available.

\section{Frequency Analysis of Three-Degree-of-Freedom Angular Motions}

Dynamic stability test techniques that permit a wind tunnel model to have three angular degrees of freedom have been shown to be essential for certain types of measurements. The hardware required for this type of testing is now available at several experimental facilities. Data reduction procedures used to analyze angular motion data frequently utilize nonlinear least-squares methods that are based on the tricyclic theory. In this theory; the motion of a slightly asymmetrical vehicle can be represented in the complex $\alpha-\beta$ plane* hy three rotating modal vecturs denuled as nutation, precession, and trim arms. The magnitudes of the nutation and precession arms are prillarlly dependent upon the initial conditions of the motion, while trim arm magnitude is related to the vehicle's nonrolling trim angle of attack.

$\therefore \alpha$ and $\beta$ are the angles of attack and sideslip, respectively, of the vehicle. 
The least-squares data reduction method requires accurate initial estimates of the angular frequencies of the three arms if the solution is to converge satisfactorily. The trim arm is generally much smaller than the other arms, and it may be impossible to estimate the frequency of this arm by visual examination of the data. In most cases, it is necessary to fit the two larger arms with the least-squares method and examine the residuals to detect the presence of a trim arm. This time consuming approach can be eliminated by using the discrete Fourier transform to estimate the spectrum of the data. The spectrum will immediately show whether the data requires epicyclic (two arm) or tricyclic analysis and accurately provides the initial frequency eștimates for the least-squares solution. When the motion is precessing very slowly and the nutation and precession arms have angular frequencies that are nearly equal, the digital spectrum may not be capable of resolving the two frequencies. However, assuming that the spectrum resolution is reasonable, the single frequency displayed in the spectrum can be used as a satisfactory initial approximation for both arms in this case. To illustrate this technique, a typical tricyclic motion was analyzed. An $\alpha-\beta$ plot of a few cycles of the simulated motion is presented in Figure 12a. Characteristic parameters of the motion are listed in the figure and are representative of data obtained during a hypersonic test of a conical vehicle. The amplitude spectrum of the data is shown in Figure 12b. In all of the applications, the spectrum will be presented with angular frequency, $\omega=2 \pi f$, as the independent variable. The dependent variable will be the DFT magnitude, $|G(j)|$. As was mentioned earlier, several factors prevent $|G(j)|$ from accurately representing the peak magnitudes of the continuous transform. Therefore, no scale is given for the spectrum ordinate. In Figure $12 b$ and succeeding figures, adjacent values of $|G(j)|$ are connected for clarity. However, it should be remembered that these are plots of discrete values, not continuous functions. Although only 3.5 cycles of data are shown in Figure 12a, 137 cycles were sampled for the spectrum calculation. 


$$
\begin{array}{lcccc}
\multicolumn{1}{c}{\text { ARM }} & \text { MAGNITUDE, DEG } & \text { FREQUENCY, RAD/SEC } & \text { DAMPING, } 1 / \text { SEC } \\
\cline { 2 - 3 } \text { NUTATION } & 3.0 & 16.0 & -0.05 \\
\text { PRECESSION } & 2.3 & -12.0 & -0.05 \\
\text { TRIM } & 0.1 & 20.0 & --.
\end{array}
$$

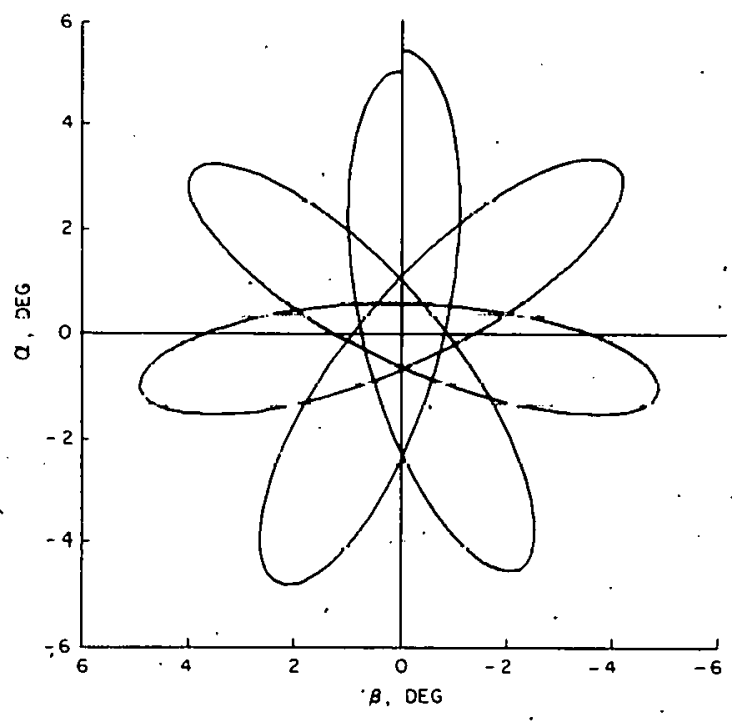

a. Motion pattern

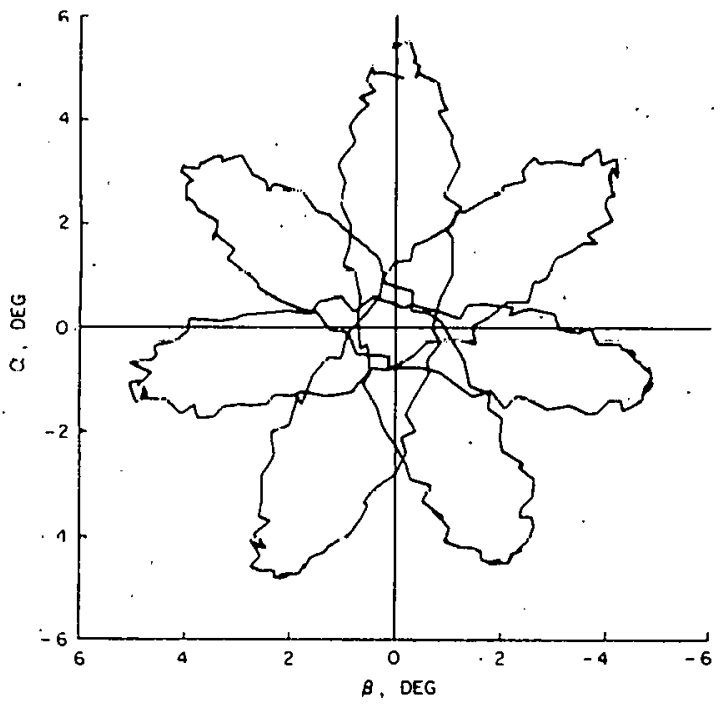

c. Motion pattern with random noise

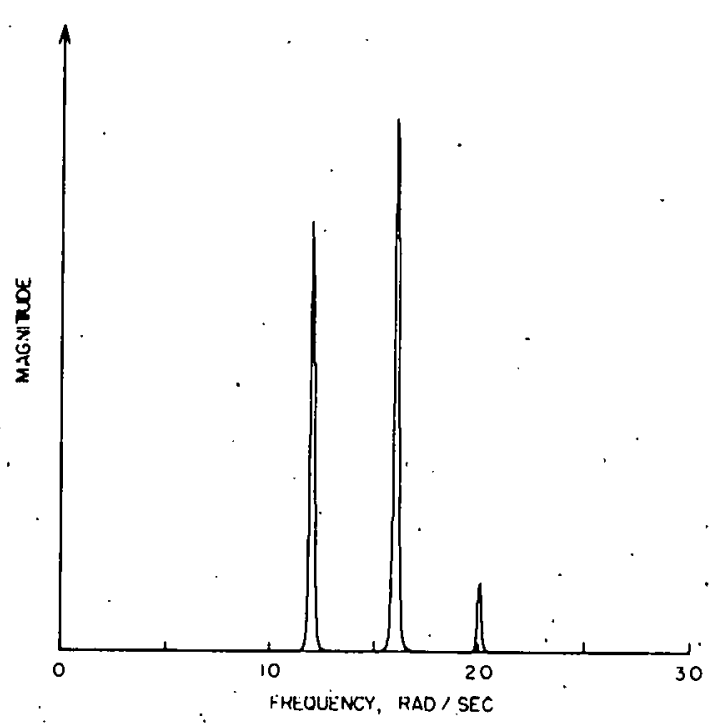

b. Amplitude spectrum

Figure 12. Simulated tricyclic motion data

48 
The sampling interval was $0.03 \mathrm{sec}$ and 2048 samples were transformed, giving a spectrum resolution of $0.1 \mathrm{rad} / \mathrm{sec}$. The Hanning window was used to reduce leakage, and aliasing was insignificant since the folding frequency is 104.7 $\mathrm{rad} / \mathrm{sec}$. The amplitude spectra of the $\alpha$ and $\beta$ data sets were.calculated separately and then averaged to give the spectrum shown in the figure.

The tricyclic theory treats the $\alpha$ and $\beta$ components of the motion as the real and imaginary parts of a complex number, $\alpha+i \beta$. Therefore, instead of averaging the spectra from two sets of real data, the spectrum could have been calculated directly from the single set of complex data. The spectrum of a set of complex data can be confusing to the casual user because the spectrum for negative frequencies is not a mirror image of that for positive frequencies, as is the case with real data. Furthermore, negative frequencies are usually displayed as folded above the positive frequencies. However, the spectra' of complex data do distinguish between positive and negative frequencies, that is, the direction of vector rotation in a complex plot (Argand diägram), and in this example the precession arm frequency would appear as negative in the spectrum. This can be a useful feature in analyzing the data and eliminates the resolution problem mentioned earlier for nearly equal nutation and precession frequencies.

Although the magnitude of the trim arm is only one-thirtieth that of the largest arm, and is not noticeable in the $\alpha-\beta$ plot, it is easily detected in the spectrum. For this application, the amplitude spectrum has been used, instead of the power spectrum, to avoid undue distortion of the spectrum magnitudes. If the power spectrum had been used, the trim arm would have been difficult to detect.

Angular motion data usually contain Gaussian noise. In Figure 12c the motion of Figure $12 \mathrm{a}$ is reproduced with random noise (standard deviation $=0.1 \mathrm{deg}$ ) added to both the $\alpha$ and $\beta$ components. Although the noise amplitude is as large as the trim arm, it does not significantly change the spectrum (Figure 12d) because the frequency content of the noise is 
spread uniformly over the entire spectrum.

\section{Elimination of Extraneous Frequencies in Dynamic Stability Data}

One technique for determining the large-amplitude dynamic stability characteristics of a high-fineness-ratio body requires that the model be supported by a slender strap which spans the wind tunnel from floor to celling. The support incorporates a one-degree-of-freedom bearing which permits the model to oscillate freely in the vertical (pitch) plane. An angular transducer provides direct output of model angle of attack as a function of time.

A series of tests were conducted in a low speed wind tunnel using a support of this type. When finned models were tested, with the fins in an $\mathrm{x}$-position to avoid interference from the strut wake, the test results were satisfactory: The amplitude-time data were undistorted and the spectrum of the data showed a single frequency, the model's aerodynamic frequency. However, attempts to test a configuration with a base flare were not as successful. Interference between the strut wake and the flare caused the model to have a small-amplitude yawing motion. The angular transducer was sensitive to both pitch and yaw motions, and the output signal was a composite wave. A typical angle of attack history for the flared configuration is presented in Figure 13a. The data have a badly distorted wave shape and could not be used to calculate accurate aerodynamic damping coefficients. It was obvious that the data had to be filtered to ejiminate the yaw component.

In order to accurately specify the digital filter characteristics, it was necessary to determine the frequency content of the data. As is the case with many composite waves, visual inspection of the waveform did not yield accurate frequency information. Therefore, digital spectra were calculated for representative sets of data. It was known that the aerodynamic frequency of the model was less than 15. rad/sec. Thus, an analog filter 

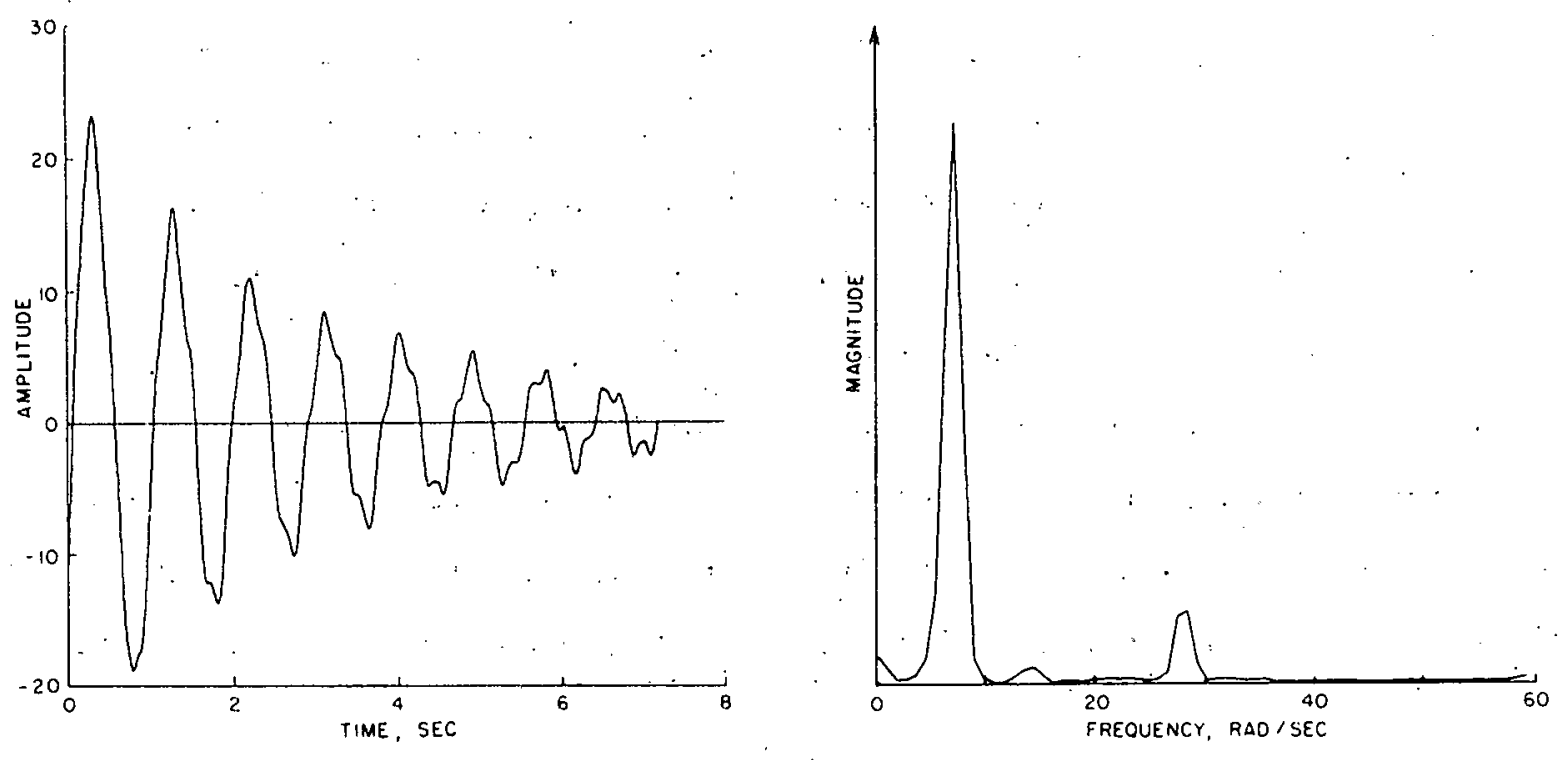

a. Angular transducer output before low-pass digital filtering.

b. Amplitude spectrum before low-pass digital filtering
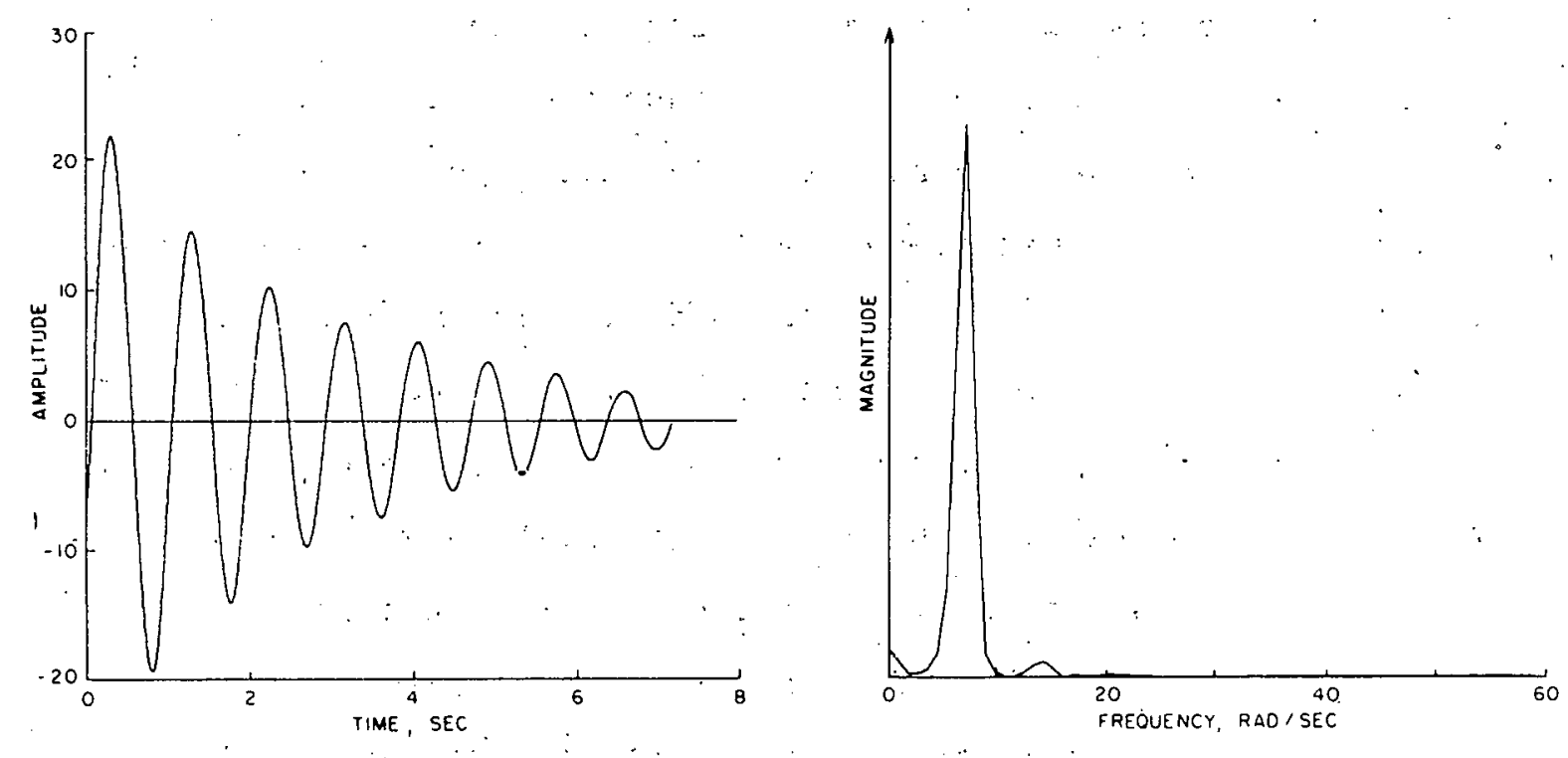

c. Angular transducer output after

d. Amplitude spectrum after low-pass digital filtering

Figure 13. Dynamic stability data with support interference 
with a cutoff frequency of $62.8 \mathrm{rad} / \mathrm{sec}$ could be used to prefilter the data without loss of basic information. The data were sampled to provide 1024 samples with a sample interval of $0.007 \mathrm{sec}$. The folding frequency is 448.8 $\mathrm{rad} / \mathrm{sec}$, so aliasing was insignificant. In this example, the sampling interval was selected to give at least 60 points per cycle at the aerodynamic frequency for subsequent use in a dynamic stability data reduction program. If only the spectrum were required, a larger sampling interval could have been used with fewer samples. However, for this set of data, the resulution of the spectrum, $0.88 \mathrm{rad} / \mathrm{sec}$, is fixed hy the data time epan, 7.2 oec, and cannot be changed by varying the sample interval or number of samples. The Hannting daca window was applied to the sampled data, and amplitude spectra were calculated.

'l'he amplitude spectrum for the data of. Figure $13 \mathrm{a}$ is presented in figure 13b. The digital spectrum should always be examined over the entire frequency range, that is, zero to the folding frequency. In this case, there was no detectable frequency. content for frequencies greater than $60 \mathrm{rad} / \mathrm{sec}$, and the frequency scale was expanded for slarity. Two eignificant frequencies are apparent. The lower frequency $(7 \mathrm{rad} / \mathrm{sec})$ is the aerodynamic frequency of the model from the unrestrained pitching motion. The yawing motion of the model, restrained by the torsional stiffness of the support system, introduces the higher frequency (28 rar/sec). Approximately eight cycles of data were sampled at the aerodynamic frequency. Equation (14) indicates that the relative accuracy of the spectrum at this frequency is approximately \pm 12 percent, which is adequate for the present application. Lxamination ul spectra from representative test runs indicated that the yaw component could be eliminated by using a digital low-pass filter with a cutoff frequency of $16 \mathrm{rad} / \mathrm{sec}$ and a termination frequency of $28 \mathrm{rad} / \mathrm{sec}$. It would have been very difficult to obtain an electronic filter having the desired frequency characteristics. If the data frequencies had changed significantly between test runs, as a result of changes in test conditions or 
model properties, the filter characteristics easily could have been changed to match the new conditions. The angular motion data after digital filtering are shown in Figure 13c. These data are suitable for analysis with conventional dynamic stability data reduction techniques. The spectrum of the filtered data is presented in Figure 13d. The high-frequency component has been attenuated, while the low-frequency information is unaffected. By the use of these techniques it was possible to obtain usable data from all of the test runs.

\section{Determination of Control Surface Forcing Function Frequencies}

Lifting surfaces, such as canards or wings, may create unsteady. disturbances that will influence the flow over control surfaces located aft of the lifting surfaces. To evaluate the control system stability, it may be necessary to experimentally determine the frequencies of the forcing functions which act on the control surface.

A test of this type was conducted at a supersonic Mach number. To obtain the desired.information, the control surface was supported on an internal control surface balance that measured the hinge moment. The entire model, including control surface and control surface balance, was supported on a six-component internal balance. The control surface balance output was recorded with an analog tape recorder. The desired bandwidth of the sampled data was $2500 \mathrm{rad} / \mathrm{sec}$, which requires a sampling interval of $0.0012 \mathrm{sec}$. This exceeded the capability of the data acquisition system, so the playback speed of the analog recorder was set at one-fourth the record speed to reduce the frequencies by a factor of four. A sampling interval of $0.004 \mathrm{sec}$ could then be used, giving the sampled data a bandwidth of $785 \mathrm{rad} / \mathrm{sec}$ which corresponds to a bandwidth of $3142 \mathrm{rad} / \mathrm{sec}$ for the actual data. During the sampling operation, the data were prefiltered with an electronic low-pass filter to attenuate frequencies greater than $628 \mathrm{rad} / \mathrm{sec}$ in the sampled data or $2513 \mathrm{rad} / \mathrm{sec}$ in the actual data. (All subsequent references to frequencies will be in terms of actual frequencies, that is, four times the sampled 
frequencies).

To establish the dynamic characteristics of the system, air-off free oscillation data were taken. These data were obtained by manually deflecting and releasing the control surface. Typical air-off data are shown in Figure $14 \mathrm{a}$ where, for clarity, only 20 percent of the 2048 samples used'in the spectral calculations are plotted. The data were adjusted to have a zero mean, and the Hanning window was applied. The digital spectrum is presented in Figure 14b where the frequency resolution is $3.1 \mathrm{rad} / \mathrm{sec}$. No significant frequency content was detected for frequencies greater than $1200 \mathrm{rad} / \mathrm{sec}$. The amplitude spectrum is presented here to avoid loss of low-magnitude frequency components. However, the power spectrum may be more appropriate for this application since it represents power input to the control system. The largest magnitude in the spectrum occurs at the natural frequency (715 $\mathrm{rad} / \mathrm{sec}$ ) of the control surface-control surface balance combination. The model was supported in a tank below the operating tunnel while these data were taken, and the frequencies adjacent to the control surface natural frequency are probably the resile of a forced osclilacton of the model by tunnel vibration. If the power spectrum were calculated, these sma11magnitude components would be insignificant." Ihe cluster of lower frequencies (50 to $120 \mathrm{rad} / \mathrm{sec}$ ) results from the natural frequencies of the model and six-component balance combination: The spike at $377 \mathrm{rad} / \mathrm{sec}$ represents $60 \mathrm{~Hz}$ noise which was present during data acquisition. Noise $(60 \mathrm{~Hz})$ introduced during data sampling appeared in the spectrum at 4 x $377=1508 \mathrm{rad} / \mathrm{sec}$ but wảs of̉ ınșignıticañnt mágnıtude.

There are two reasons for presenting this example: (1) to demonstrate that digital spectra can be used to accurately determine the frequencies present in a complicated waveform; and (2) to show how a narrow frequency region can be isolated by using digital filters. In this application, the $60 \mathrm{~Hz}$ noise was easily eliminated through the use of a digital band-reject (notch) filter. The filter had a unit gain for frequencies less than 


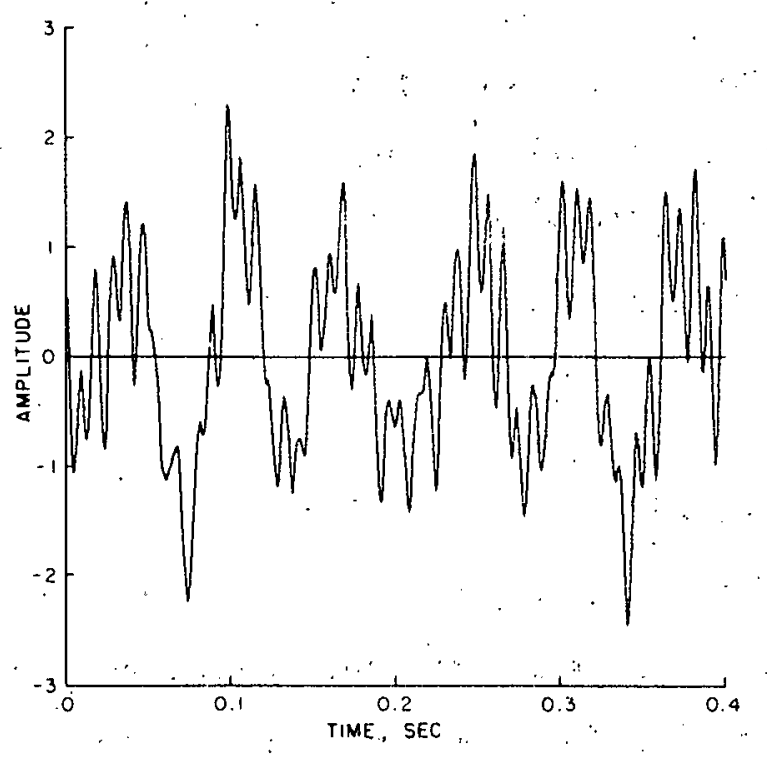

a. Control balance output before band-reject digital filtering

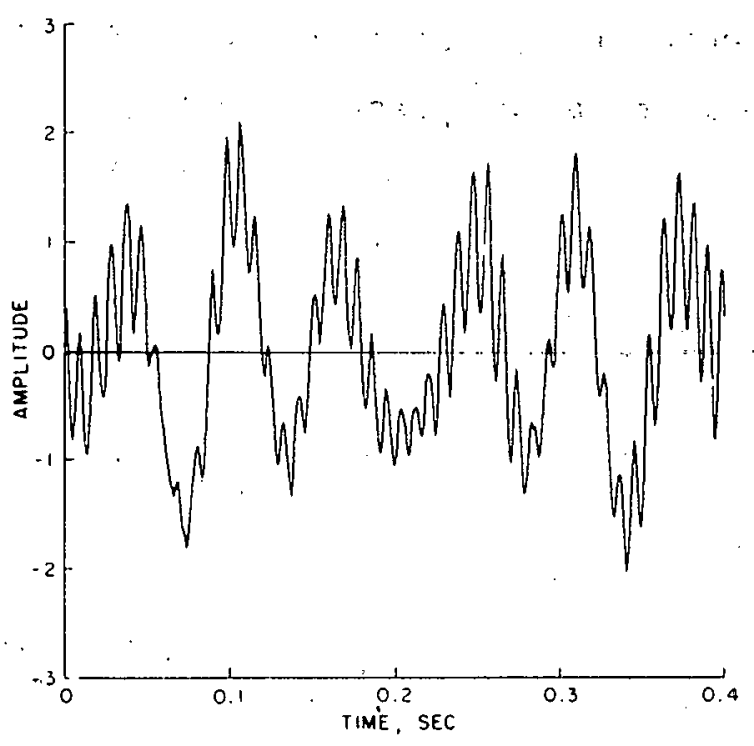

c. Control balance output after band-reject digital filtering

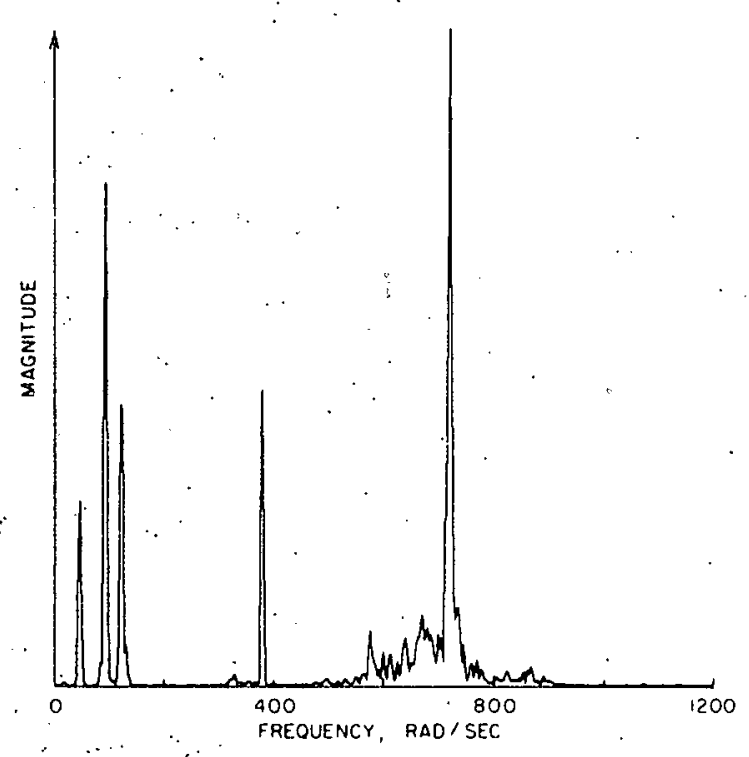

b. Amplitude spectrum before : band-reject digital filtering

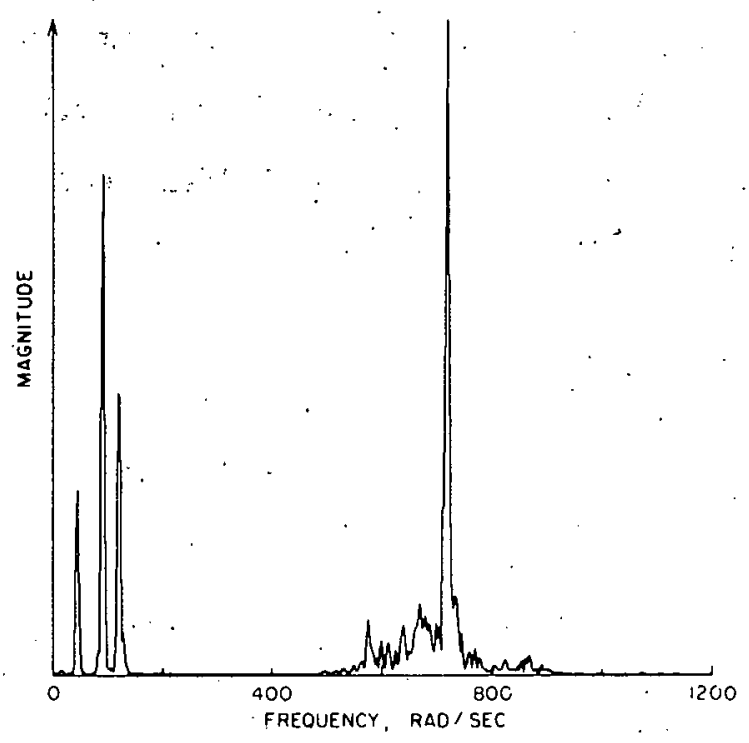

d. Amplitude spectrum after band-reject digital filtering

Figure 14. Control surface dynamics 
$200 \mathrm{rad} / \mathrm{sec}$, zero gain for 340 to $420 \mathrm{rad} / \mathrm{sec}$, and unit gain for frequencies greater than $560 \mathrm{rad} / \mathrm{sec}$. The filtered data and associated spectrum are shown in Figures $14 \mathrm{c}$ and 14d. None of the frequency content outside of the rejection region has been changed by the filtering operation. In a similar manner, a narrow frequency region can be retained while frequencies outside the region are attenuated. This is accomplished by using a digital bandpass filter.

\section{Concluding Remarks}

It has been shown that digital spectral analysis and filtering techniques can be useful tools in aerodynamic testing. Examples have been used to demonstrate that the frequency content of time-dependent data can be determined and specified frequency regions can be isolated or attenuated. Although it is impossible to completely cover the field of digital signal processing in a report of this type, the information presented provides a usable introduction to the subject. 


\section{References}

1. Brigham, E. O., The Fast Fourier Transform, Prètice-Ha11, Inc., Englewood Cliffs, N. J., 1974, p. 23.

2. Cochran, W. T., et al,, (G-AE Subcommittè on Measurement Concepts), "What is the Fast Fourier Transform?" IEEE Transactions on Audio and Electroacoustics, Vol. AU-15, No. 2, June 1967, pp. 45-55.

3. Rabiner, L. R., et a1。, "Terminology in Digital Signal Processing," IEEE Transactions on Audio and Electroacoustics, Vo1. AU-20, No. 5, December 1972, pp: 322-337.

4. Brigham, op. cit., Chapters 2, 3, and 4.

5. Bracewe11, Ron, The Fourier Transform and Its Applications, McGraw-Hi11 Company, Inc., New York, 1965, Chapters 6 and 18.

6. Ibid., p. 8

7. Brigham, op. cit., p. 108 .

8. Cooley, J. W., Lewis, P. A. W., and Welch, P. D., "Historical Notes on the Fast Fourier Transform," IEEE Transactions on Audio and Electroacoustics, Vol. AU-15, No.'2, June 1967, pp. 76-79.

9. Cooley, J. W., and Tukey, J. W.; "An Algorithm for the Machine Calculation of Complex Fourier Series,.". Mathematics of Computation, Vo1. 19, No. 90, Apri1 1965, pp. 297-301.

10. Singleton, R. C., "A Short Bibliography on the Fast Fourier Transform," IEEE Transactions on Audio and Electroacoustics, Vol. AU-17, No. 2, June 1969, pp. 166-169.

11. Bergland, G. D., "A Guided Tour of the Fast Fourier Transform," IEEE Spectrum, Vo1. 6, July 1969, pp. 41-52.

12. Maynard, H. W., "An Evaluation of Ten Fast Fourier Transform (FFT) Programs," Research and Development Technical Report ECOM-5476

(AD-758 451), March 1973, U. S. Army Electronics Command, White Sands Missile Range, N. M.

13. Singleton, R. C., "An Algorithm for Computing the Mixed Radix Fast Fourier Transform," IEEE Transactions on Audio and Electroacoustics, Vol. AU-17, No. 2, June 1969, pp. 93-103.

14. Brenner, N. M., "Three Fortran Programs that Perform the Cooley-Tukey Fourier Transform," Technical Note 1967-2, July 1967, Massachusetts Institute of Technology, Lincoln Laboratory, Lexington, Mass.

15. Larson, A. G., and Singleton, K. C., "Real-time Spectral Analysis on a Small General-Purpose Computer," Proceedings of the 1967 Fall Joint Computer Conference, AFIPS, Vo1. 31, 1967, pp. 665-674.

16. Cadzow, J. A., Discrete-Time Systems, Prentice-Ha11, Englewood Cliffs, N. J., 1973, pp. 293-295. 
17. Bingham, C., Godfrey, M. D., and Tukey, J. W., "Modern Techniques of Power Spectrum Estimation," IEEE Transactions on Audio and Electroacoustics, Vo1. AU-15, No. 2, June 1967, pp. 56-66.

18. Welch, P. D., "The Use of Fast Fourier Transform for the Estimation of Power Spectra: A Method Based on Time Averaging Over Short, Modified Periodograms," IEEE Transactions on Audio and Electroacoustics, Vol. AU-15, No. 2, June 1967, pp. 70-73.

19. Muh1stein, L., Jr., and Coe, C. F., "Integration Time Rcquired to Extract Accurate Static and Dynamic Data From Transonic Wind-Tunne1 Tests," AIAA Paper No. 75-142, AIAA 13th. Aerospace Sciences Meeting, Pasadena, California, Jan. 20-22, 1975.

20. Kuo, F. F., and Kaiser, J. F., Ed., System Analys is by Digital Computer, John'Wiley \& Sons, New. York, 1966, pp. 218-285.

21. Stearns, S. D., "Digital Filter Design Routines," SLA-73-0871, October 1973, Sandia Laboratories, Albuquerque, N: M.

22. Graham, R. J., "Determination and Analysis of Numerical Smoothing Weights," NÁSA TR R-179, December 1963, George C. Marshall Space Flight Center, Huntsville, Alabama.

23. Jones, J. A., and Graham, R. J., : "DDP-116 Genera1 Digital Filtering," NASA TM X-64697, September 1972 , George $C$. Marsha11 space F1ight Center, Huntsvilie, Alabama.

24. Taylo, J. T., "Digital Filters for Non-Rea1-Time Data Processing," NASA CR-880, October 1967, Northeast"Louisiana State College, Monroe, La. 
DISTRIBUTION :

ARO, Inc. (2)

von Karman Gas Dynamics Facility

Arnold Air Force Station, TN 37389

Attn: L. L. Trimmer

J. C. Uselton

1000 G. A. Fowler

Attn: C. D. Broyles, 1100

W. A. Gardner, 1200

1111 S. D. Stearns

O. E. Jones, 1700 .

1300 D. B. Shuster

Attn: A. A. Lieber, 1310

M. L. Kramm, 1320

1313 J. R. Keisey

R. G. Clem, 1350

1330 R. C. Maydew

1331 H. R. Vaughn

1332 C. W. Peterson

1333 S. McAlees

1334 D. D. McBride

1334 D. E. Berg

1334 E. L. Clark (15)

1334 R. H. Cro11 (15)

1335 W. R. Barton

1335. W. E. Williams on

1336 J. K. Cole

1336 W. H. Curry

1400 A. Y. Pope

2642 R. E. Jones

8266 E. A. Aas (2)

3141 C. A. Pepmueller (Actg.) (5)

3151

W. L. Garner (3)

For: ERDA/TIC (Unlimited Releașe)

ERDA/TIC (25)

(R. P. Campbe11, 3171-1) 